\title{
EXAMEN CRÍTICO DE LA TÉCNICA DE TABLAS DE VIDA EN LAS TENDENCIAS SOBRE FECUNDIDAD: EL CASO DE MÉXICO
}

\author{
FÁtima JuÁrez
}

El Colegio de México

\section{INTRODUCCIÓN}

EL PROCESO DE FORMACIÓN DE familias en sus distintas cohortes, considerado a través del análisis de intervalos en los nacimientos, conlleva un estudio de la fecundidad más interesante y mejor detallado. La familia se construye en la medida en que la mujer atraviesa por distintas etapas: del matrimonio o unión al primer nacimiento, del primer nacimiento al segundo y por los intervalos de nacimientos de orden sucesivos hasta alcanzar el tamaño final de la familia. Al momento de iniciar esta investigación había sólo algunos estudios de la fecundidad, desde esta perspectiva: Young (1977) realizó un estudio sobre el proceso de formación de familias en Australia, y Pool (1978) retomó este enfoque para aplicarlo a una ciudad de Canadá. En 1979 surgieron otros dos proyectos, mi investigación ${ }^{1}$ y otro, a cargo de la World Fertility Survey, ${ }^{2}$ en el que, a diferencia

1 Mi trabajo de investigación corresponde al proyecto doctoral, utilizo datos de México y América Latina, la primera etapa de los resultados fueron presentados en la 2a. Reunión Nacional de Investigación Demográfica en México, 1980. El presente artículo forma la segunda parte de los resultados parciales, en forma preliminar.

2 Véase Hobcraft y Rodriguez (1980). 
de los trabajos anteriores utiliza datos que provienen de países en vías de desarrollo. El aparente descuido que ha habido en este tipo de análisis se debe en parte a la falta de información detallada, es decir, sobre las historias del embarazo de las mujeres, que se recaban únicamente en encuestas especiales.

La Encuesta Mexicana de Fecundidad de 1976-1977 (EMF), ${ }^{3}$ proporciona el tipo de información que se requiere para el estudio de los intervalos entre nacimientos por cohortes (grupos quinquenales de edad por cohortes de nacimientos). Dicha encuesta es un estudio de corte transversal, diseñado para calcular los niveles y tendencias en la fecundidad. Es la primera encuesta para todo el país en la que se entrevistaron 7310 mujeres, solteras y casadas, con edades que oscilaban entre 15 y 49 años, con excepción de mujeres solteras sin hijos de edades entre 15 y 19 años. Las historias del embarazo de 6255 mujeres casadas entre 15 y 49 años forman la base del estudio ${ }^{4}$ se eliminaron a las mujeres que tuvieron nacimientos premaritales. ${ }^{5}$ El análisis de intervalos entre nacimientos se referirá únicamente a los nacidos vivos.

El análisis no es directo, a pesar de la información disponible, en virtud de los problemas que ocasiona que las historias del embarazo no sean completas. Se debe otorgar un tratamiento especial a las historias truncadas de las cohortes incompletas $y$ a las interacciones entre los intervalos en los nacimientos.

Se utilizaron dos metodologías para el análisis de intervalos entre nacimientos para intentar superar estos problemas: $a$ ) la técnica de las tablas de vida, y $b$ ) el enfoque de truncamiento. El análisis de las tablas de vida es una técnica sofisticada que otorga un tratamiento adecuado al efecto censal que incluye la información recopilada en la EMF. El enfoque de truncamiento es una técnica más sencilla, que también elimina una parte de los problemas ocasionados cuando faltan completar las historias de los nacimientos. El segundo método se

3 La Encuesta Mexicana de Fecundidad forma parte de la Encuesta Mundial de Fecundidad.

4 En el texto se utilizan las mujeres casadas o en unión como sinónimo, puesto que únicamente se preguntó en la EMF la fecha de unión.

5 La proporción de mujeres con nacimientos premaritales es bastante elevada, $10.1 \%$, así como las concepciones premaritales, 12.5\%. Se decidió eliminar del análisis a las mujeres con nacimientos premaritales en virtud de que forman un grupo especial con respecto a su comportamiento de la fecundidad y con otras características sociales y demográficas. 
presenta para cuestionar la confiabilidad y utilidad de las tendencias de la fecundidad que se infieren de la técnica de las tablas de vida.

\section{Problemas en el análisis asociado con las historias DE NACIMIENTOS TRUNCADOS}

En sentido general, puede suponerse que las cohortes más viejas en las mujeres casadas comprendidas en la EMF, llegaron a completar su periodo reproductivo y las historias de embarazo que declararon en el momento de la entrevista darán razón del tamaño total de la familia. Para estas mujeres se pueden hacer cálculos en dos medidas importantes de fecundidad que se asocian con el proceso de formación de la familia: las probabilidades de crecimiento de la familia y la distribución total del intervalo de los nacimientos. Sin embargo, esto no se aplica a otras mujeres porque sus historias de nacimientos están truncadas. Esto quiere decir que algunas mujeres han alcanzado el nacimiento $i$ y el $i-n$ en la fecha de la entrevista mientras que otras no habían alcanzado el nacimiento $i$. Por ejemplo, una mujer entrevistada en la EMF casada, de 22 años y con un hijo, tiene una reducción de riesgo de exposición con respecto a las de edad más avanzada; probablemente, el paso del primer nacimiento a un posible segundo nacimiento queda interrumpido a causa de la fecha de la entrevista. Algunos demógrafos (como Ryder, Hobcraft, Rodríguez. . .) llaman a este acortamiento "efecto censal" (censoring effect).

Fecha de la entrevista
Uniön Nacimiento 1o.
$\frac{\text { Nacimiento 2o. Nacimiento 3o. }}{\text { Futura fecundidad 0, 1,2. nacimientos }}$

Es decir, no pueden calcularse de manera directa las probabilidades de crecimiento de la familia ni la distribución total de intervalos, debido a las historias de embarazo truncadas de las cohortes incompletas.

La selección de mujeres que se casan jóvenes y que son más fecundas en cuanto al efecto de selectividad de la muestra, es otro problema importante en el análisis de intervalos entre nacimientos, ocasionado también por la reducción de exposición de los diferentes grupos de edad. Así, las medidas inferidas para los diferentes grupos 
de edad no pueden ser representativas para toda la población. Ilustremos esta idea con un ejemplo.

Supongamos que queremos estudiar la transición del segundo al tercer nacimiento de la cohorte de mujeres entre 15 y 19 años. En primer lugar, las mujeres con edades entre 15 y 19 años que entraron en el análisis en la fecha de la entrevista contaban con una unión; la característica común era la edad temprana de llegar al matrimonio. Por otro lado, únicamente entraron en el análisis las mujeres que tenían uno o dos descendientes. Así, las cohortes entre 15 y 19 años de edad están integradas por mujeres que se casaron a temprana edad y que tuvieron dos hijos, relativamente rápido. Disminuirá la intensidad del efecto de selectividad en la medida en que aumente la edad; por ejemplo, para cohortes de más edad. Cuando del mismo modo se analizan los intervalos en los nacimientos de las mujeres algu na vez casadas de las cohortes de 15 a 19 años, éstas habrán llegado en una edad temprana al matrimonio y será más fecunda que el promedio de las mujeres que cuenta con edades de 20 a 24 años.

\section{Metodología}

Se escogieron dos métodos para el análisis de los intervalos entre nacimientos: $a$ ) la técnica de las tablas de vida, y b) el enfoque de truncamiento.

\section{Técnica de las tablas de vida}

La técnica de las tablas de vida, que por lo general se ùtiliza en la mortalidad, es una herramienta poderosa de análisis. No existen muchas aplicaciones de ellas en el estudio de la formación de la familia. Christabel M. Young utilizó esta técnica en Australia en 1977 para analizar el espaciamiento de los hijos y los patrones de cambio en el periodo de procreación (Encuesta de la familia en Melboume). Janel Pool también utilizó tablas de vida en 1978 para estudiar el espacia. miento y escalonamiento entre nacimientos en una región industrializada; Hobcraft y Rodríguez la aplicaron a un país en vías de desarrollo (1980, para la Encuesta de Colombia que forma parte de la Encuesta Mundial de Fecundidad).

La técnica de las tablas de vida considera adecuadamente a los grupos de personas que están expuestas al riesgo, pero que no han 
alcanzado todavía el evento. En los intervalos de nacimientos se elimina el sesgo que se introduce a causa de la fecha de la entrevista en la exposición al riesgo, es decir, se elimina el efecto censal además de manejar la información disponible de manera muy completa. Por otra parte, la distorsión que introduce el efecto de selectividad puede eliminarse parcialmente por medio de controles adecuados.

La aplicación de este método, a pesar de sus ventajas, solamente resulta significativa si la calidad de la información es relativamente buena. La valoración de la EMF, llevada a cabo por M. Ordorica y J. Potter (1980), muestra que la información es relativamente buena y que, por lo tanto, resulta conveniente aplicar la técnica de las tablas de vida para efectuar el análisis de los intervalos entre nacimientos.

La técnica se aplica por cohortes, a cada intervalo de las distin. tas etapas de la construcción de familias:

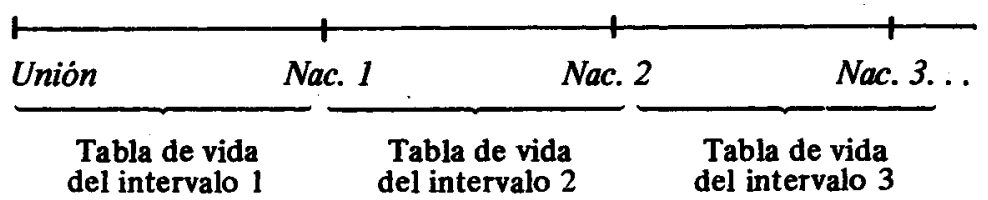

Intervalo 1: de la unión al primer nacimiento

Intervalo 2: del primero al segundo nacimiento

Intervalo 3: del segundo al tercer nacimiento

$$
\text { : }
$$

Intervalo 10: del noveno al décimo nacimiento

La duración de la exposición es cero en el comienzo de cada intervalo. Las medidas que se obtienen representan las probabilidades condicionales de la transición de la etapa $i$ al $i+1$, denotado $B^{i}+{ }^{1}$ (donde $i+1$ se refiere al intervalo). Así, para el primer intervalo se obtiene la probabilidad condicional de que cada vez que la mujer está casada, tendrá su primer nacimiento $\left(B^{1}\right)$; y para el siguiente intervalo se obtiene una probabilidad condicional acumulada de tener un nacimiento $i+1$ dado que la mujer ha tenido el $i$ nacimiento $\left(B^{i}+{ }^{1}\right)$ (véase el apéndice para la deducción de la fórmula).

El tiempo de exposición de cada intervalo puede medirse en meses o años; pero las probábilidades condicionales calculadas resultarán más precisas si los cálculos de Bi se refieren a duraciones cortas. La duración que se escogió para la construcción de las tablas de vida fue de dos meses y un año, y la diferencia entre ambas en este caso es estadísticamente despreciable. 
La información requerida para la aplicación de la técnica de tablas de vida consiste en cuadros de doble entrada del número de mujeres en distinta duración de exposición $\left(N_{x}\right)$-cada mes o año y su status terminal- lo que se alcance primero, el evento $\left(E_{X}\right)$ o la entrevista $\left(C_{x}\right)$ por cohortes para cada intervalo.

De las tablas de vida se obtienen dos tipos de medidas para cuantificar el proceso de formación de familias. La proporción de mujeres con un nacimiento $i$ que pasan al nacimiento $i+1$ en un periodo $x$ $\left(B_{x}^{i}\right)$, mide la intensidad de la fecundidad. $Y$ la mediana del intervalo entre nacimientos $(M)^{6}$ que se refiere a espaciamiento de la fecundidad, es decir, al tiempo que le toma a la mitad de las mujeres pasar de una paridez a otra. ${ }^{7}$

\section{Enfoque de truncamiento}

Este segundo enfoque es un método más simple que Ryder sugirió en 1974. Se basa en el truncamiento de la experiencia de las varias cohortes por edades específicas de modo que puedan compararse directamente. Nuestro interés principal es comparar la formación de familias de distintas cohortes y examinar si se han llevado a cabo algunos cambios en el espaciamiento y calendario de los nacimientos que conduzcan a cambios en la fecundidad. En virtud de que las historias de los nacimientos están incompletas, no es posible comparar el patrón de fecundidad de los distintos grupos de edad puesto que las dos cohortes sucesivas de cinco años - cohorte $(x, x+5)$ y cohorte $(x+5, x+10)$ no han alcanzado el final del periodo reproductivo $y$ han sido expuestas a diferentes periodos.

Si se cortaran los últimos cinco años de experiencia de la cohorte $(x+5, x+10)$-cohorte indicada como $(x+5, x+10)^{t_{-}}$, es decir, cuando estas mujeres tenían la edad de $(x, x+5)$, sería posible compararla directamente con la cohorte $(x, x+5)$. En nuestro análisis, cada uno de los grupos quinquenales de edad $C_{x}, x+5$ está truncado 5 años y se compara con la cohorte quinquenal adyacente más joven.

6 También se calculó la medida $T=\left(q_{1}+2 q_{2}+q_{3}\right) / 4$ (Tukey, 1977) que sugirieron Hobcraft y Rodríguez (1980, EMF) como una medida de ubicación más sensible. Sin embargo, como el examen de los resultados que se obtuvieron con esta medida son muy similares a la mediana, en el presente estudio se escogió al último para el análisis.

7 Referencia por Hobcraft y Rodríguez como el tempo y quantum de la fecundidad. 
Ejemplo: en el diagrama "a" para la experiencia de mujeres entre 30 y 35 años de edad a la fecha de la entrevista $\left(C_{30-35}\right)$ se trunca hasta la edad 25-29 $\left(C_{30-35}\right)^{t}$ y se compara con la experiencia de mujeres de edades 25.29 a la fecha de la entrevista $\left(C_{25-29}\right)$. De manera similar, en el diagrama " $b$ ", la experiencia de las mujeres que al momento de la encuesta tenían 25 y 29 años, se recorta hasta la edad 20-24 $\left(C_{25-29}\right)^{t}$, to que hace posible comparar su experiencia con la de la cohorte de 20-24 $\left(C_{20-24}\right)$...

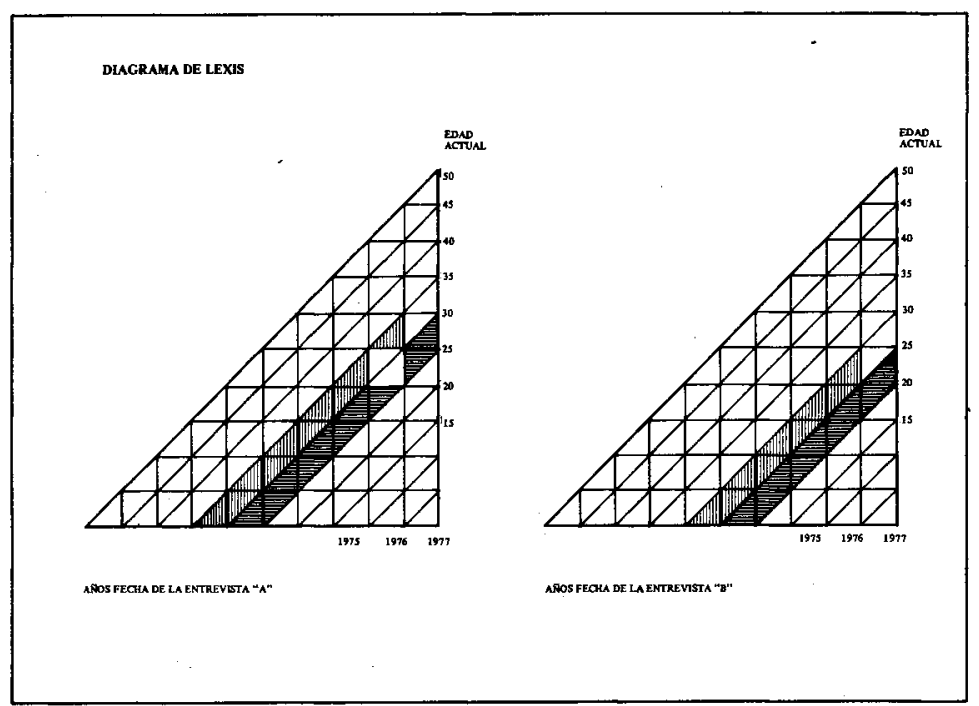

Para este enfoque de truncamiento se han calculado dos tipos de medidas, la proporción de mujeres con $n$ nacimientos que continúan a $(n+1)$ y el intervalo promedio. Debe notarse que este método estudia únicamente los intervalos cerrados de los nacimientos.

Este enfoque más simple nos permite tratar con el problema ocasionado por el efecto censal y además, en parte, elimina el sesgo originado por el efecto de selectividad. 
EXAMEN DE LA TÉCNICA DE TABLAS DE VIDA PARA LAS TENDENCIAS EN FECUNDIDAD

Las tablas de vida proporcionan una serie de probabilidades acumulables de duraciones distintas de exposición, para cada intervalo entre nacimientos. Hobcraft y Rodríguez (1980) encontraron en la EMF para Colombia que la proporción de mujeres que tenía un nacimiento de un orden particular dentro de los cinco años siguientes al nacimiento previo, proporciona un magnifico indicador del nivel de fecundidad cuando se utilizan las historias retrospectivas de las mujeres que aún no han completado su periodo de reproducción final. Estas probabilidades acumulables de una duración de exposición de cinco años $B_{60}$ es un periodo lo suficientemente grande para el suceso del siguiente evento, $y$ es una aproximación a las probabilidades de crecimiento de las familias. Por otra parte, si el análisis de $\boldsymbol{B}_{60}$ no muestra ningún cambio, al comparar las distintas cohortes, se puede analizar otra duración de exposición: $1,2,3 \circ 4$ años $\left(B_{12}, B_{24}\right.$, $B_{36}, B_{48}$ ).

La intensidad de la fecundidad que aparece en el intervalo del primer nacimiento es más fuerte para las mujeres más jóvenes; $88 \%$ de las mujeres de edades de $45-49$ años tienen su primer nacimiento dentro de los cinco primeros años posteriores a la fecha de unión, lo que significa $92 \%$ para las cohortes de $40-44$ años de edad. Este porcentaje sigue incrementando hasta 96 y $97 \%$ en mujeres de edad actual de 20-24 y 15-19 años respectivamente (véase gráfica A.1, cuadro 1).

Al referirnos al calendario del primer nacimiento, la mediana del

Cuadro 1. Probabilidades acumuladas de 60 meses de exposición al riesgo en el primer intervalo del proceso de formación de la familia para mujeres alguna vez unidas, por cohortes.

\begin{tabular}{|c|c|c|c|c|c|c|c|}
\hline \multirow{2}{*}{$\begin{array}{l}\text { De la } \\
\text { unión al } \\
\text { nac. } 10 .\end{array}$} & \multicolumn{7}{|c|}{ Edades de las mujeres en la fecha de la entrevista } \\
\hline & $15-19$ & $20-24$ & $25-29$ & $30-34$ & 35-39 & $40-44$ & $45-49$ \\
\hline $\begin{array}{c}\mathrm{B}_{60} \\
\text { Mediana }\end{array}$ & $\begin{array}{r}9656 \\
14.53\end{array}$ & $\begin{array}{l}.9613 \\
14.51\end{array}$ & $\begin{array}{l}.9559 \\
13.81\end{array}$ & $\begin{array}{l}9396 \\
14.10\end{array}$ & $\begin{array}{l}.9334 \\
15.35\end{array}$ & $\begin{array}{l}.9220 \\
16.03\end{array}$ & $\begin{array}{l}.8839 \\
16.73\end{array}$ \\
\hline
\end{tabular}

Fuente: Cuadro A-1 
intervalo se reduce en la medida en que decrece la edad hasta llegar a las mujeres que contaban con una edad de 20-24 años, grupo de edad debajo del cual el espaciamiento del primer intervalo aumenta ligeramente, es decir, las mujeres más jóvenes están retardando sus nacimientos.

Surgen resultados similares para los intervalos siguientes, una tendencia de aumentar la fecundidad para cohortes más jóvenes con excepción de la cohorte de edades 40-44 años en el noveno intervalo, que muestra un descenso en la fecundidad (de 80 a 78\%). También observamos que los niveles de fecundidad son muy elevados: $85 \%$ de las mujeres de 30-39 años que han tenido un sexto nacimiento continúan, al séptimo nacimiento, dentro de los cinco años siguientes $B_{60}^{7}$, y más de $80 \%$ de las mujeres que tenfan un octavo hijo continúan, al noveno, dentro de un periodo de cinco años $B_{60}^{9}$. (Véase gráfica A-1 y cuadro 2 .)

En lo que se refiere al espaciamiento de los hijos medido por la mediana del intervalo, la gráfica A.2, -cuadro A.1- muestra que por lo general las mujeres tienden a tener sus niños más rápidamente en la medida en que desciende la edad, en especial a partir del sexto intervalo.

De los valores anteriormente presentados se puede concluir que la tendencia de las probabilidades acumuladas $B_{60}$ es engañosa. Es poco probable que esté aumentando la fecundidad en mujeres más jóvenes. ${ }^{8}$ Aunque es cierto que la técnica de las tablas de vida elimina el sesgo que introdujo la reducción de la exposición en la fecha de la entrevista -efecto censal- sin embargo no elimina el efecto de selectividad. Se han sobreestimado las probabilidades para las cohortes más jóvenes en virtud de que se refieren a mujeres de transición rápida por las diferentes etapas del proceso de formación de la familia y este efecto es mayor para mujeres más jóvenes. Es por ello que el aumento en la intensidad de la fecundidad, es especial en edades jóvenes, lo que no significa que exista un aumento en la fecundidad puesto que se han sobreestimado los valores de los índices y aquellos

8 En sentido estricto y en virtud de que la historia del embarazo de las mujeres está truncada por la fecha de la entrevista, no podemos hablar en un aumento o descenso en la fecundidad hasta que las mujeres hayan terminado su periodo reproductivo. Sin embargo, es muy probable que si observamos periodos prolongados de exposición -por ejemplo $B_{60}-$ estos cambios se asocien con cambios en la fecundidad. 
en los que sí hay indicios de un descenso de la fecundidad han disminuido. Así, cuando se comparan dos cohortes adyacentes, si existe un descenso en $B_{x}^{i}$ para el grupo más jóven, se observa que las tendencias se encaminan en la dirección adecuada aun cuando estén subestimadas.

Como nuestro interés se centra en los cambios ocurridos dentro de el proceso de formación de familias y, en especial, en la transición

Cuadro 2. Probabilidades acumuladas de 60 meses de exposición de riesgo de las diferentes etapas del proceso de formación de la familia para mujeres alguna vez unidas, por cohortes.

\begin{tabular}{lllllll}
\hline $\begin{array}{l}\text { B60 } \\
\text { Intervalo } \\
\text { de naci- } \\
\text { mientos }\end{array}$ & $20-40$ & $25-29$ & $30-34$ & $35-39$ & $40-44$ & $45-49$ \\
\hline 10. a 20. & .9408 & .9289 & .9187 & .9288 & .9016 & .8835 \\
20. a 30. & & .9095 & .8869 & .9069 & .8746 & .8785 \\
30. a 40. & & .8756 & .8730 & .8689 & .8858 & .8705 \\
40. a 5o. & & & .8547 & .8662 & .8739 & .8330 \\
50. a 60. & & & .8913 & .8588 & .8422 & .8014 \\
60. a 70. & & & .8564 & .8504 & .8299 & .8278 \\
7o.a 80. & & & .8993 & .8320 & .8085 & .7973 \\
80. a 90. & & & .8877 & .8807 & .7789 & .7994 \\
90. a 10. & & & & .8114 & .7758 & .6855 \\
\hline
\end{tabular}

Fuente: Cuadro A.1.

hacia un descenso de la fecundidad, es posible buscar exclusivamente los indicadores que señalan dichas modificaciones y así obtener el panorama del descenso; las probabilidades estarán subestimadas pero mostrarán la tendencia correcta. El examen de las $B_{60}$ 's no muestra ningún descenso en la fecundidad por lo que se pasará a analizar las probabilidades acumuladas de menor duración. Se eliminarán del análisis a las cohortes cuyas tablas de vida cuentan con pocas mujeres expuestas a riesgos en el comienzo del intervalo y a los grupos extremos que se ven muy afectados por la selectividad. ${ }^{9}$

9 Únicamente se analizará la información de mujeres entre 20 y 49 años 
Quisiéramos hacer algunos comentarios sobre las mujeres casadas de edad avanzada antes de continuar con el análisis de las probabilidades acumuladas de duración más corta.

La cohorte de mujeres más viejas ( $45-49$ años) presenta probabilidades de crecimiento más bajas en la transición del primer al segundo nacimiento así como en otros intervalos que las que se observan en las mujeres adyacentes más jóvenes. Resultado contrario al que se esperaría en virtud de que las mujeres de edad más avanzada ya están alcanzando el final de su periodo reproductivo y tal vez el tamaño final de su familia. Esto es, $\boldsymbol{B}_{60}$ muestra un movimiento más acelerado de la cohorte de edades $40-44$ en los primeros intervalos que el de las mujeres en edades de 45-49. Este cambio puede significar un verdadero aumento de la fecundidad en cohortes de 40-44 años en relación con las cohortes de 45-49 años, o puede ser el reflejo de un error en el reporte de los nacimientos y la fecundidad pudo haberse mantenido constante.

Brass y otro estudioso demostraron cómo un error en la historia del embarazo puede distorsionar el nivel y la tendencia de la fecundidad. Se han detectado dos errores en la ubicación de los eventos en el tiempo: ${ }^{10}$ el primero se relaciona con el tamaño del periodo de referencia y el segundo con la localización del periodo de referencia. El primer tipo de error surge en todas las mujeres mientras que el segundo tiende a emerger más en las mujeres de mayor edad porque ubican erróneamente sus nacimientos, los atrasan (Brass, 1977), o bien, los adelantan (Potter, 1977). No es nuestra intención determinar la existencia de este tipo de errores en la información de la EMF, ni tampoco señalar la forma en que afecta nuestro nivel de fecundidad; sin embargo, queremos comentar que es muy probable que los niveles de fecundidad más bajos en el grupo de edades $45-49$ estén distorsionados por los errores en el informe de los nacimientos. Estudios similares en muchos países han demostrado la existencia de tales desviaciones.

de edad para el primero y segundo intervalos; para el tercero y cuarto intervalos solamente a las que tienen de 25 a 49 años, y a partir del cuarto intervalo, a mujeres de 30 o más años.

$10 \mathrm{Se}$ han hecho esfuerzos por resolver este problema. Se han desarrollado diversos modelos como el Gompertz transformado (Booth, 1979) y el Modelo Gompertz Relacional (Zaba, 1979) con el fin de detectar y corregir estos errores. 
La gráfica A.1 -cuadro A.1-, muestra las probabilidades acumuladas de progresion al siguiente nacimiento para diversas duraciones. El examen de la duración de exposición bajo cinco años muestra un patrón de espaciamiento más prolongado para las cohortes más jovenes que muy probablemente podría asociarse con un descenso en la fecundidad.

Para el primero y segundo intervalos, las mujeres que contaban con 20-24 años fueron las únicas que mostraron un descenso en la probabilidad de alcanzar el primero y segundo nacimientos. Esto puede interpretarse como un mayor espaciamiento entre nacimientos $\left(\begin{array}{lll}B_{12} & \text { y } & B_{24}\end{array}\right)$ : estos cambios se subestimaron por causa del efecto de selectividad. En el tercer intervalo, las mujeres entre 30-34 años, en la fecha de la entrevista, muestran un pequeño descenso en la fecundidad si se las compara con las de 35-39 años. A partir del sexto nacimiento, la inclinación de selección es tan fuerte que parece que los niveles de fecundidad aumentan con el tiempo.

Cuadro 3. Resumen de los cambios ocurridos en el proceso de formación de familias orientadas a un descenso.

Descenso en la intensidad de la fecundidad según cohortes quinquenales

\begin{tabular}{llllll}
\hline Intervalo 1 & de & $\mathbf{C}(25-29)$ & $\mathbf{a}$ & $\mathbf{C}(20-24)$ & $\mathbf{B}_{36}, \mathbf{B}_{24}, \mathbf{B}_{12}$ \\
Intervalo 2 & de & $\mathbf{C}(25-29)$ & $\mathrm{a}$ & $\mathbf{C}(20-24)$ & $\mathbf{B}_{48}, \mathbf{B}_{36}, \mathbf{B}_{24}$ \\
Intervalo 3 & de & $\mathbf{C}(35-39$ & $\mathrm{a}$ & $\mathbf{C}(30-34)$ & $\mathbf{B}_{60}, \mathbf{B}_{48}, \mathbf{B}_{36}$ \\
Intervalo 5 & de & $\mathbf{C}(35-39)$ & a & $\mathbf{C}(30-34)$ & $\mathbf{B}_{60} \mathbf{B}_{48}, \mathbf{B}_{36}, \mathrm{~B}_{24}$
\end{tabular}

Fuente: Cuadro A.1.

\section{ELIMINACIÓN DEL EFECTO DE SELECTIVIDAD}

Como ya observamos, cuando se analizan las probabilidades acumuladas $B_{x}^{i}$, surgen varios problemas para lograr la interpretación. Este efecto de selectividad se lleva a cabo de dos maneras: $a$ ) la información para cohortes más jovenes proviene de mujeres que se casaron jóvenes y $b$ ) cuando se considera la exposición al riesgo, solamente las mujeres que se casaron muy jóvenes pueden contribuir con exposiciones prolongadas (es decir, a paridez elevada). La combinación 
de estos dos efectos tiende a incrementar las probabilidades calculadas $B_{\dot{x}}^{i}$

El efecto de un cambio en la edad del casamiento es otro elemento que debe tomarse en consideración. El aumento en la edad del casamiento tendería a disminuir la probabilidad de las mujeres que van de la paridez $n$ a la $n+1$ por causa de una reducción en la exposición al riesgo.

Estos problemas -en especial el del efecto de selectividad que ocasiona resultados con desviaciones- pueden en parte superarse si se homogeneizan dos distintas cohortes de tal manera que las mujeres que se casan jóvenes y son más fecundas formen un grupo distinto al de las que se casan en edades más avanzadas. Esto puede lograrse al controlar las distintas edades en el matrimonio. Por ejemplo, para el primer intervalo podrían compararse las probabilidades acumuladas de crecimiento de la familia $\left(B_{x}^{i}\right)$ para cohortes de mujeres que se casan a la edad de 16 años, o las que casan a la edad de 17, y así sucesivamente. Para el segundo intervalo, podemos controlar tanto la edad del matrimonio como la edad al primer nacimiento, que son los factores que afectan la incidencia y espaciamiento del segundo nacimiento. Se escogió la edad del nacimiento anterior para controlar todos los intervalos de nacimientos. Hobcraft y Rodríguez (1980) muestran que la edad en los nacimientos previos -que mencionaremos como edad relativa ${ }^{11}$ es un control demográfico eficiente, "consistente, interpretable y que tal vez (muestra) diferencias sustanciales".

La dificultad para depurar el control de los grupos es una limitante en este procedimiento. No es posible controlar la edad relativa año con año puesto que el número absoluto de mujeres que están expuestas al riesgo en cađa tabla de vida sería muy pequeño. Por esta razón se decidió homogeneizar las cohortes controlando para cuatro grupos de edad el inicio de cada intervalo. Estos se dividieron según los cuartiles de los intervalos con el objeto de obtener una distribución similar de mujeres en cada grupo: ler. grupo, mujeres con edades menor que el primer cuartil $\left(<Q_{1}\right)$; 20 . grupo, mujeres con edades entre el primero y el segundo cuartil $\left(Q_{1} \leqslant Q_{2}\right)$, 3er. grupo, mujeres con edades entre el segundo y el tercer cuartil $\left(Q_{2} \leqslant Q_{3}\right)$, y 40 . grupo, mujeres con edades igual o más avanzadas que el tercer cuartil $\left(\geqslant Q_{3}\right)$. El cuadro A.3 muestra los grupos control de edad relativa: se calcularon 320 tablas de vida para lograr el efecto de selectividad.

11 Ryder (1973), Stoto y Menken (1977) se refieren a ésta como la edad .relativa. 
Se ilustra este nuevo procedimiento de control de la edad relativa con un ejemplo (véase cuadro 4). Si en el cuarto intervalo se comparan las mujeres de edades de 25-29 años con las de 30-34 $B_{60}$ no muestra ningún cambio, mientras que después de aplicar los controles $B_{60}$ indica un descenso, la intensidad disminuye y, en conjunto, parece que las mujeres están retardando sus nacimientos.

Cuadro 4. Ilustración sobre el procedimiento de control de la edad relativa para el cuarto intervalo.

4o. intervalo: Transición del tercero al cuarto nacimiento

\begin{tabular}{|c|c|c|c|c|}
\hline \multirow[t]{2}{*}{ Medidas } & \multicolumn{2}{|c|}{ Cohorte (25-29) } & \multicolumn{2}{|c|}{ Cohorte $(30-34)$} \\
\hline & $\mathbf{B}_{60}$ & (M) & $B_{60}$ & (M) \\
\hline $\begin{array}{l}\text { Grupo no controlado } \\
\text { Grupo controlado }\end{array}$ & .8756 & (26.7) & .8730 & $(26.5)$ \\
\hline $\begin{aligned} & \leqslant Q_{1} \\
Q_{1} & \leqslant Q_{2} \\
Q_{2} & \leqslant Q_{3} \\
& \geqslant Q_{3}\end{aligned}$ & $\begin{array}{c}.9208 \\
.9301 \\
.8253 \\
-\end{array}$ & $\begin{array}{l}(22.3) \\
(27.0) \\
(26.6) \\
(36.4)\end{array}$ & $\begin{array}{l}.9630 \\
.9536 \\
.8857 \\
.7648\end{array}$ & $\begin{array}{l}(24.7) \\
(23.2) \\
(25.7) \\
(30.6)\end{array}$ \\
\hline
\end{tabular}

Fuente: Cuadro A.2.

El examen de las probabilidades de crecimiento de la familia para los cuatro grupos control en cada uno de los intervalos de nacimientos indican que existe un cambio dirigido hacia el descenso en la fecundidad. Con el objeto de presentar de manera simple los cambios observados en estos resultados, los cuatro conjuntos de control se agruparon en uno, mismos que aparecen en las gráficas A.3 y A.4 -cuadro A.2-.

Cuando se controla la edad relativa en el primer y segundo intervalo se observa con mayor claridad la tendencia hacia el descenso de la fecundidad en las mujeres más jóvenes. Sin embargo, el patrón es similar que el presentado en la gráfica A.1 (sin controlar el efecto de selectividad para los otros intervalos). Los únicos cambios que conducen al descenso en la fecundidad son indicados en el cuadro 5 :

Con el análisis de las técnicas de tablas de vida puede concluirse que la fecundidad en México ha permanecido casi constante y que el cambio es sorprendentemente lento. Esto puede ser ocasionado por el efecto de selectividad que aún afecta nuestros resultados, puesto que existe evidencia, proveniente de otras fuentes de información, 
Cuadro 5. Resumen de los cambios ocurridos en el proceso de formación de familias orientados a un descenso, cuando se controla la edad relativa de la mujer.

Descenso en la intensidad de la fecundidad según cohortes quinquenales

\begin{tabular}{llllllll}
\hline Intervalo 1 & de & $\mathrm{C}(25-29)$ & a & $\mathrm{C}(20-24)$ & & & \\
Intervalo 2 & de & $\mathrm{C}(25-29)$ & a & $\mathrm{C}(20-24)$ & & & \\
Intervalo 4 & de & $\mathrm{C}(30-34)$ & a & $\mathrm{C}(25-29)$ & & & \\
Intervalo 5 & de & $\mathrm{C}(40-44)$ & a & $\mathrm{C}(35-39)$ & a & $\mathrm{C}(30-34)$ & a \\
& & & & & & $\mathrm{C}(25-39)$ & \\
Intervalo 6 & de & $\mathrm{C}(35-39)$ & a & $\mathrm{C}(30-34)$ & a & $\mathrm{C}(25-29)$ & \\
\hline
\end{tabular}

Fuente: Cuadro A.2.

de que la fecundidad está disminuyendo y no precisamente a un paso tan lento.

\section{UN MÉTODO ALTERNO}

No existe duda de que hasta este momento la técnica de las tablas de vida es el mejor método para analizar la información censada. Pero como hemos observado, el análisis de intervalos en los nacimientos de las historias de nacimientos truncados produce un sesgo que está ocasionado por grupos de movimiento rápido. Se aplica un segundo método, basándonos en un procedimiento de truncamiento como un intento por obtener resultados similares a los de la técnica de las tablas de vida, aunque de una manera más sencilla. La idea que reside detrás es encontrar las tendencias en la fecundidad y tomar en consideración el efecto de selectividad y los probiemas que ocasionan la experiencia truncada de las mujeres.

Los índices que se obtuvieron son medidas burdas de la intensidad de la fecundidad y el calendario de los nacimientos: la proporción de las mujeres que continúa el nacimiento $n$ al $n+1$-con base en información sobre los intervalos cerrados- y el promedio de longitud de los intervalos. Aunque estas medidas tienen algunas limitaciones, resultan adecuadas para nuestros objetivos. La gráfica A.5 -cuadro A.4 y A.5- muestra estos resultados. 
En el primer intervalo se hará una comparación de las cohortes adyacentes, por ejemplo, la cohorte de 25.29 años con la experiencia de la cohorte de 30-34 truncada hasta la edad de 25-29 años ( $\left.C-\frac{\text { - }}{6} 0^{-3} \cdot 34\right)$. Encontramos que la proporción de mujeres de edades $25-29$ años que van de la unión al primer nacimiento es de $93 \%$; sin embargo, dicha proporción aumenta a $96 \%$ en el mismo intervalo para mujeres que en la actualidad tienen de 30-34 antos cuando tenían la edad de 25-29 años. Así que existe $3 \%$ de descenso en la intensidad de la fecundidad cuando se compara el cohorte de 25-29 y el de 30-34, y una reducción del intervalo promedio. El análisis se hace de manera similar para cada una de las cohortes quinquenales adyacentes en cada intervalo. La gráfica A.5 -cuadro A.4 - presenta estos niveles comparativos de fecundidad. La gráfica A.5 se dibujará como una curva continua a modo de ilustrar el análisis por cohortes de la proporción de mujeres que van del nacimiento $n$ al $n+i$.

Los fragmentos de línea de la gráfica A.5 presentan las tendencias de la fecundidad y las diferenciales que se derivaron a través del método de truncamiento. Este enfoque nos proporciona una visión de los cambios que están efectuándose en México en cuanto a fecundidad se refiere. La proporción de mujeres, que van de la unión al primer nacimiento y del primero al segundo nacimiento, permanece constante para el primero y segundo intervalos, excepción dada de mujeres más jóvenes en donde se presenta un descenso en la intensidad, acompañado por un intervalo promedio más prolongado, el que a largo plazo tendrá un efecto en el nivel de fecundidad. Se observa mejor esta tendencia que se dirige hacia un descenso en la fecundidad a partir del tercer intervalo, excepción dada del décimo intervalo. Por ejemplo, la proporción de las mujeres de 30-34 años de edad que continúan de un segundo a un tercer nacimiento es más reducida $(87.8 \%$ ), si se la compara con el $91.4 \%$ del cohorte de $35-39$ años truncado hacia las edades de 30-34 años ( $C t_{35-39}$ ); el intervalo promedio aumenta ligeramente de 26 meses para $\mathrm{Ct}_{35-39}$ a 27 meses para $C_{30-34}$ (cuadros A.4 y A.5). Se observan resultados similares para las mujeres más jóvenes.

El cambio en el proceso de formación de familias que conduce a un descenso en la fecundidad se observa claramente en la transicion del segundo al tercer nacimiento, del tercero al cuarto nacimiento y del cuarto al quinto nacimiento, donde la proporción de mujeres que van de una etapa a la siguiente desciende con la edad; el intervalo promedio global aumenta ligeramente. Parece que podemos concluir que el cuadro general, en cuanto a las tendencias de la fecundidad y 
a las diferenciales que se derivan del enfoque de truncamiento, es una representación sensible de la realidad del comportamiento de la fecundidad en México.

\section{Comparación de LOS dos métodos}

Las tendencias de la fecundidad y las diferenciales que se obtuvieron a través de la técnica de las tablas de vida tienen variantes en relación con las que se obtuvieron utilizando el enfoque de truncamiento.

Como ya mencionamos, la proporción de mujeres que van del nacimiento $i$ al $i+I$ dentro de los siguientes cinco años ( $B_{b 0}$ sin control), utilizando la técnica de las tablas de vida, arrojaron una desviación en los resultados a causa del efecto de selectividad, y $B \dot{b} 0=$ que controla la edad en el nacimiento anterior es (hasta este momento) un cálculo más refinado ya que este último elimina una parte del efecto de selectividad. La comparación del patrón de fecundidad derivado, utilizando el enfoque de truncamiento, prueba que controlando la edad en los nacimientos anteriores no se elimina de manera significativa el efecto de selectividad y, por tanto, los resultados que se obtienen aún están sesgados. Así, la tendencia que se observa es engañosa, a pesar de que se ha aceptado que la edad en el nacimiento precedente es un buen control para abordar el problema de la selectividad. La gráfica A.6 muestra el patrón de fecundidad que se obtuvo con los dos métodos: la técnica de tablas de vida con edad controlada en el nacimiento previo y el enfoque de truncamiento.

El enfoque de truncamiento indica un descenso en la fecundidad en el tiempo, a partir del tercer nacimiento mientras que el patrón obtenido a través de la técnica de las tablas de vida indica un descenso casi imperceptible en la fecundidad - la tendencia real se ve disminuida por el efecto de selectividad. Il enfoque de truncamiento arroja resultados más coherentes y parece ser que las $p^{i}$, s no se ven afectadas de manera significativa por el efecto de selectividad. En sentido general, podríamos decir que las probabilidades acumuladas $B{ }_{60} \cdot$ nos dan el nivel de la curva y el enfoque del truncamiento su forma.

\section{COMENTARIOS Finales}

El análisis del intervalo en los nacimientos nos permite examinar en detalle el proceso de formación de familias al medir la intensidad de 
la fecundidad y el espaciamiento en los nacimientos. El problema que surge cuando se trata con historias de nacimientos es el impedimento en la comparación directa de los cohortes. Se aplicó la técnica de las tablas de vida para el intervalo en los nacimientos al estudio de las tendencias y diferencias en la fecundidad. Las medidas derivadas para cada paridez son las probabilidades acumuladas de las mujeres que tienen un nacimiento después de cierto espacio de tiempo $B_{x}^{i}$ y el tiempo que le toma a una media mujer pasar de un estadio al otro. Este método aborda con éxito la información censal. Por desgracia, el efecto de selectividad afecta el análisis del intervalo de los nacimientos y las probabilidades acumuladas tienden a sobreestimarse en mujeres más jóvenes; consecuentemente muestran una tendencia errónea en la fecundidad, excepción dada de los cohortes más jóvenes en el primero y segundo intervalos, en donde la fecundidad muestra un ligero descenso.

Fue posible eliminar, después de controlar la "edad relativa", una parte del efecto de selectividad. En el cuarto intervalo se llevó a cabo un descenso en las probabilidades acumuladas del crecimiento de la familia y un incremento de la media del intervalo para cohortes que tenían menos de 30 años. Sucedió lo mismo para el quinto y sexto nacimientos para las cohortes que tenían menos de 45 y 40 años, respectivamente; es decir, se ha confirmado un cambio aún en las cohortes más viejas de mujeres alguna vez unidas (Juárez, 1980). No nos sorprende encontrar un descenso en la fecundidad de la mujer mexicana sino que el cambio sea tan lento.

El segundo método, el del truncamiento, atractivo por su sencillez, se utilizó para obtener una estimación no refinada del proceso de formación de familias. Este método presenta una tendencia más clara en el tiempo del descenso en la fecundidad que se da a partir del tercer nacimiento, excepción dada en el décimo intervalo. Los patrones de cambio que se calcularon con este método muestran diferencias más fuertes en el descenso de la fecundidad y parecen ser más coincidentes con la realidad mexicana. Este hallazgo nos lleva a concluir que a pesar del esfuerzo que se hizo por eliminar el efecto de selectividad en el análisis en que se utilizaron las tablas de vida, los resultados que se obtuvieron aún se ven afectados por sesgos (de selectividad).

Para terminar, sólo queremos reafirmar la utilidad general de la técnica de las tablas de vida en el análisis del intervalo de los nacimientos pero se debe hacer hincapié en la necesidad de buscar caminos que lleven a eliminar las desviaciones y sesgos en los cálculos sobre 
fecundidad. Además, también se requiere hacer investigación para encontrar otros métodos alternos, tal vez técnicas sencillas como el enfoque de truncamiento aquí presentado, que traten satisfactoriamente el problema de las historias incompletas de los embarazos y que al mismo tiempo nos proporcionen resultados válidos, como en el caso de la técnica de las tablas de vida. 


\section{Apéndice}

\section{CONSTRUCCIÓN DE UNA TABLA DE VIDA}

DE DECREMENTO SENCILLO

Resulta adecuado utilizar la técnica de tablas de vida si: $a$ ) la duración de la exposición es central en los casos del evento en estudio; $b$ ) si puede medirse la duración, y $c$ ) si el evento es único y no es ambiguo (Smith, 1980).

En nuestro caso, los eventos en estudio satisfacen estas condiciones: a) la duración de exposición al riesgo es fundamental para el caso del nacimiento; $b$ ) puede medirse la duración en meses, años, etcétera, por ejemplo, tener un primer nacimiento después de un año, dos años, ... de exposición, y c) el evento es único para la mayoría de los casos, porque existe solamente un primer nacimiento, un segundo nacimiento, y así sucesivamente, excepción dada de los nacimientos múltiples. Con el fin de adaptar nuestro evento a esta tercera condición, pueden eliminarse los nacimientos múltiples y contarse como en orden de nacimientos diferentes con intervalos cero - como en nuestro caso.

$C_{x}$ La información que se requiere para cada tabla de vida es $N_{x}$, ${ }^{C} C_{x}$ y $n E_{x}$ 
$N_{x}$ es el número total de las mujeres expuestas al riesgo al comienzo del intervalo; $n C_{x}$ es el número total de casos censales -entrevistados- en el intervalo $x$ a $x+n$; si el evento y la entrevista se llevan a cabo durante la misma duración del intervalo se considerará el evento como censado y se incluyen en ${ }_{n} C_{n} ; y_{n} E_{x}$ es el número de eventos en el intervalo $x$ a $x+n$

$$
\begin{gathered}
N_{x+n}=N_{x}-{ }_{n} C_{X}-{ }_{n} E_{x} \\
N_{n}^{*}=N_{x}-\frac{1}{2}{ }_{n} C_{x}
\end{gathered}
$$

donde $\mathrm{N}_{\mathrm{x}}^{*}$ es el número corregido de mujeres que estuvieron expuestas al riesgo.

La proporción de mujeres que van del nacimiento $i$ al $i+1$ $(i=0,1,2, \ldots)$ en un periodo determinado. es decir, la probabilidad condicional para alcanzar el siguiente nacimiento en una duración $\mathrm{x}$, es:

$$
\begin{gathered}
B_{x}=1-n^{b} x \cdot n^{q_{x} \text { donde }} \\
n^{q_{x}}=\frac{N^{E} E_{X}}{N n}
\end{gathered}
$$

es la proporción de mujeres que van del nacimiento $i$ al $i-1$ (probabilidad no condicional), y $\boldsymbol{n}_{x}$ es la proporción de mujeres que, con respecto del total, tienen el nacimiento $i+1$ en el intervalo $x$ al $x+n$.

Se supone como punto de partida que $n_{0}=B_{n}=n^{q}$ en virtud de que las mujeres expuestas al riesgo de duración cero con un nacimiento $i$ no han alcanzado el nacimiento $i+1$, y la probabilidad acumulada para la duración $x+n$ es $B_{x+n}=B_{x}+n^{b} x$

Ver a Hobcraft y Rodríguez (1980, EMF) para un ejemplo detallado de la construcción de la tabla de vida del intervalo en los nacimientos, y para un enfoque teórico del análisis de tablas de vida, referirse a Sheps y Menken (1976) y a Hoem (1970). 


\section{GRAFICA A.1}

Probabilidades acumuladas ( $\mathrm{Bx}^{i}$ ) del intervalo de los nacimientos para mujeres alguna vez unidas según duracion de exposición al riesgo $12,24,36,48$ y 60 meses, por cohortes.
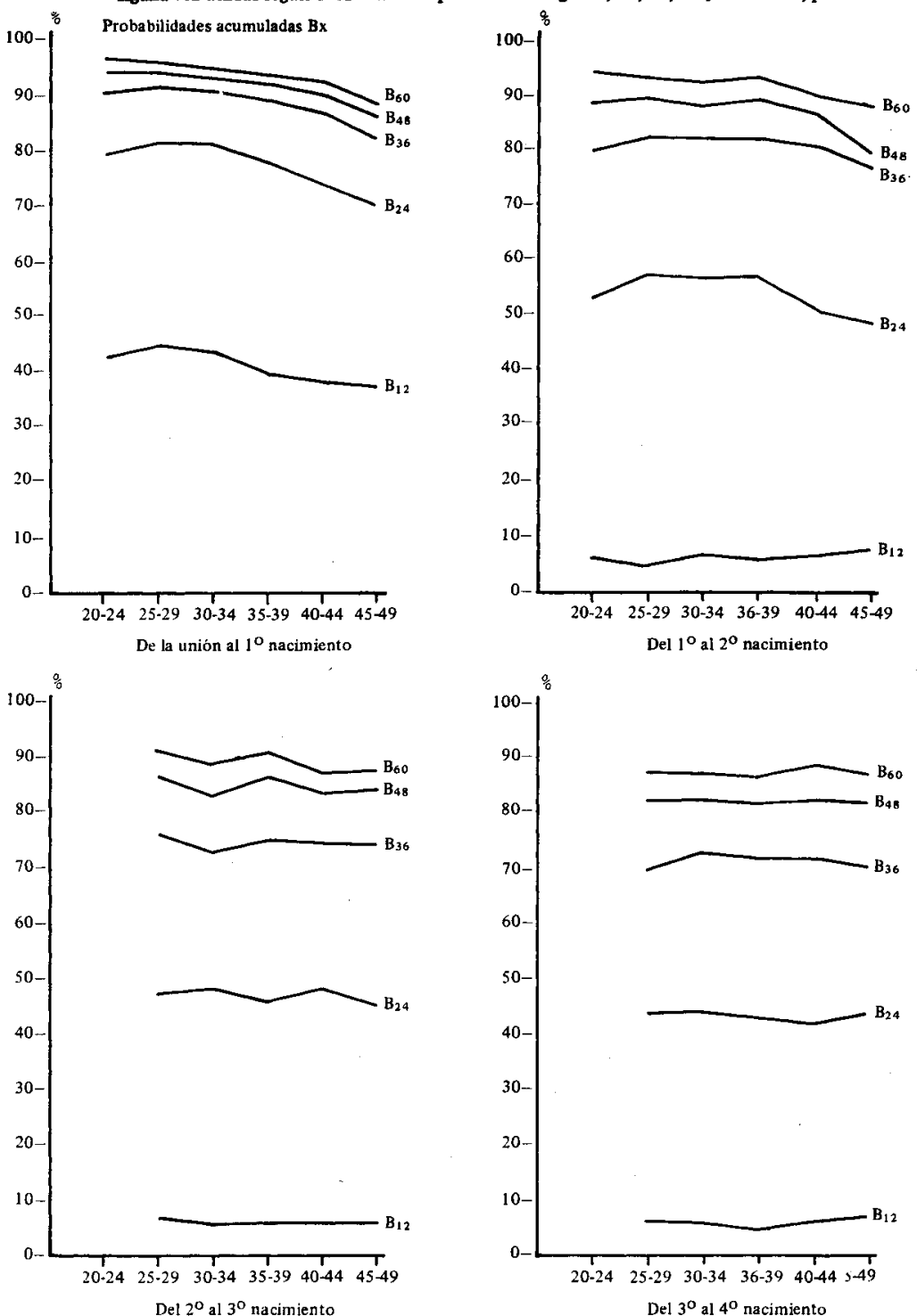
Del $1^{\circ}$ al $2^{\circ}$ nacimiento

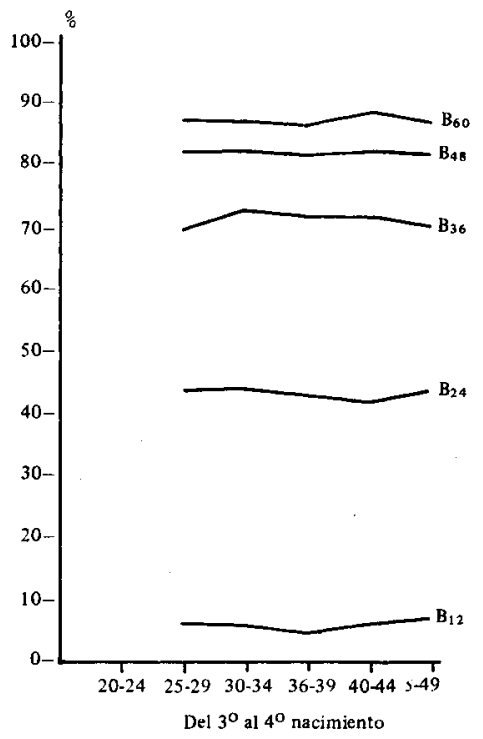


GRÁFICA A-1 (Continuación)
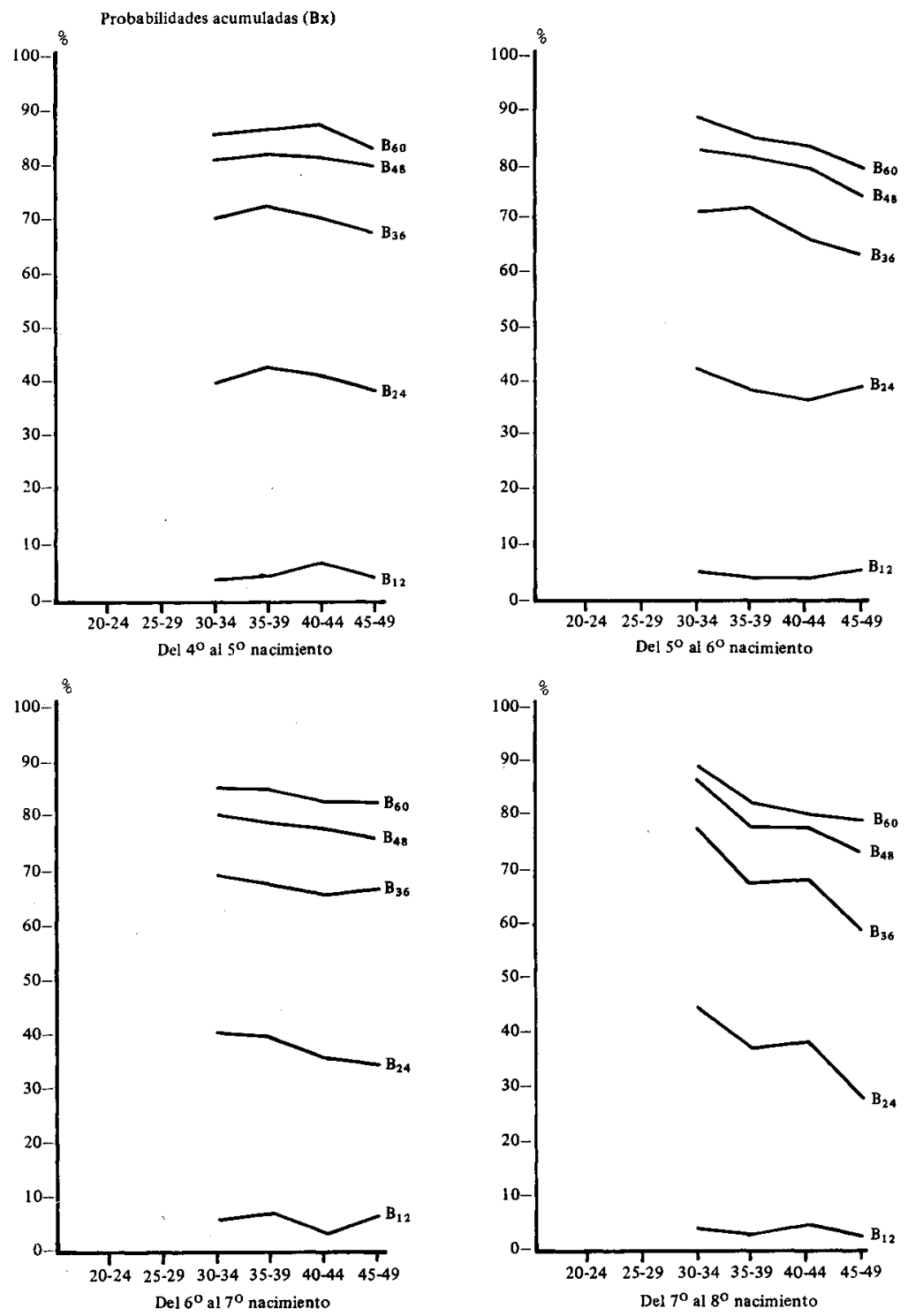

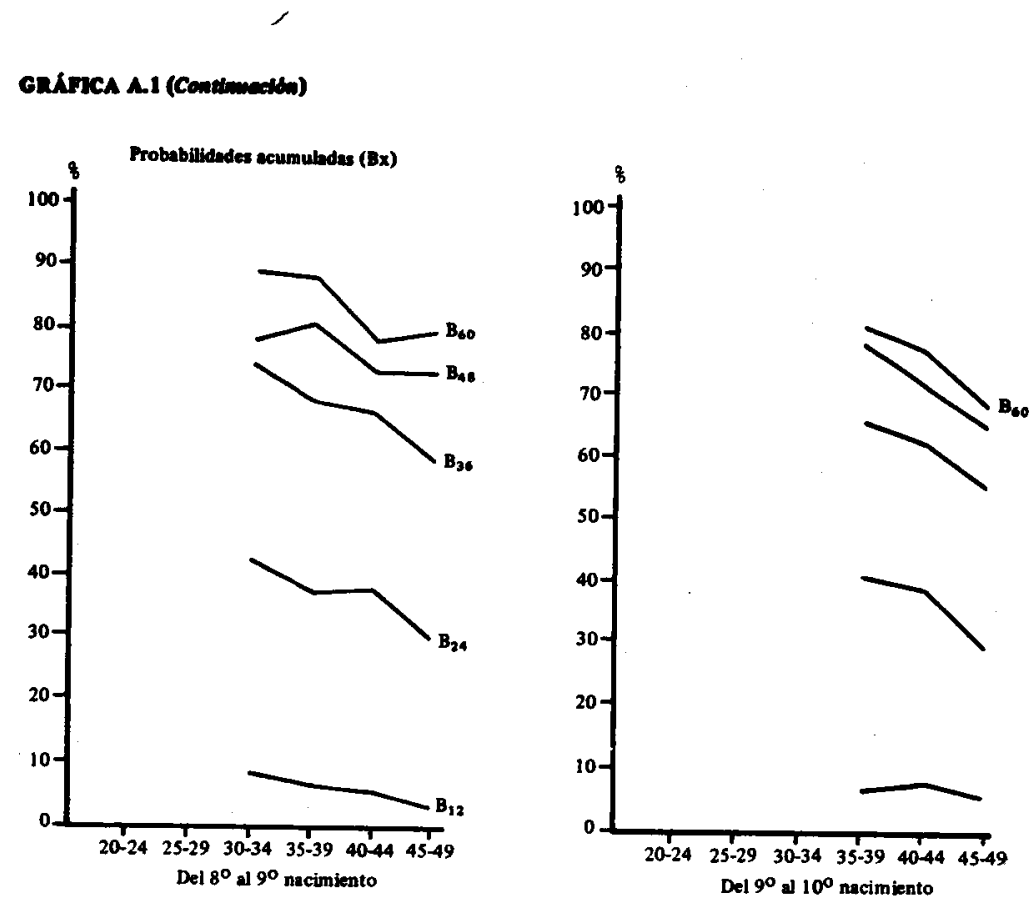

Fuente: Cuadro A.1 
Mediana en:
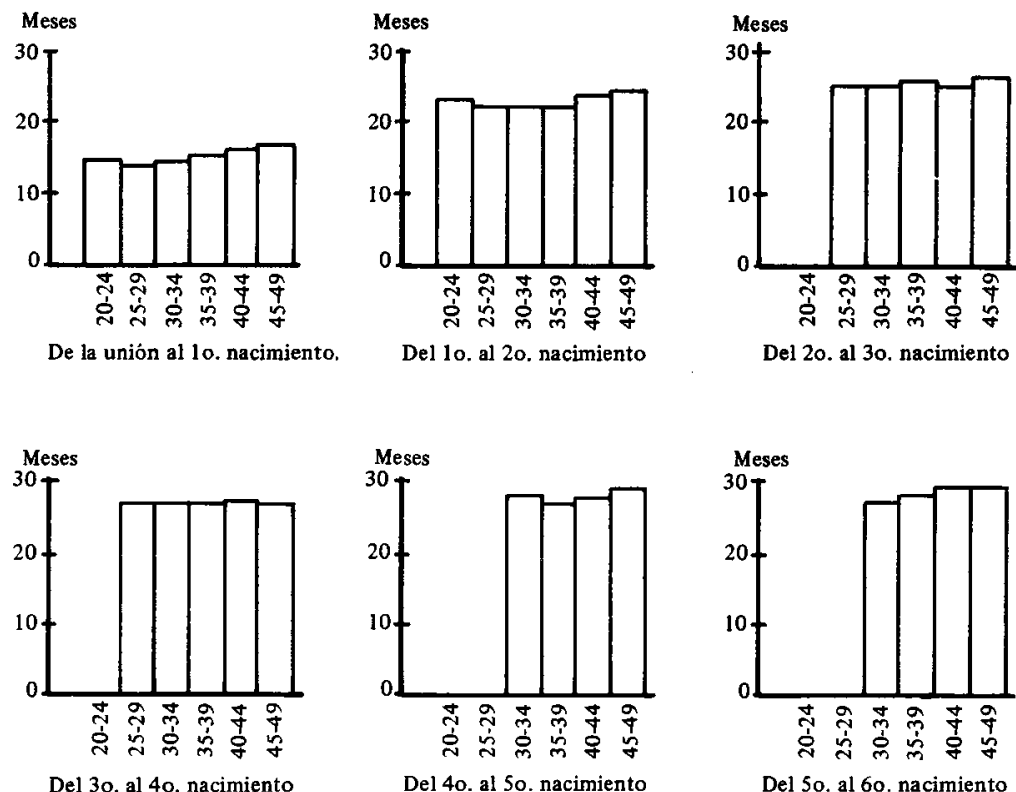

Del 3o. al 4o. nacimiento

Del 4o. al So. nacimiento

Del So. al 6o. nacimiento
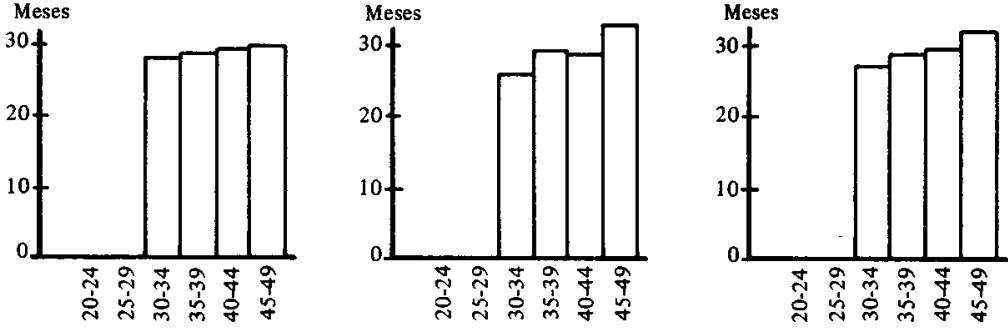

Del 7o. al 80 . nacimiento

Del 8o. al 9o. nacimiento

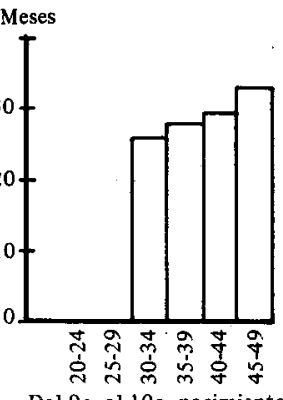

Fuente: Cuadro A.1 


\section{GRAFICA A.3}

Probabilidades acumuladas $\left(\mathrm{Bx}^{1}\right)$ del intervalo de los nacimientos controlando ta edad de las mujeres en el nacimiento previo pera mujeres algun vez unidas segun duracion de exposición al riesgo $12,24,36,48$ y 60 meses por cohortes
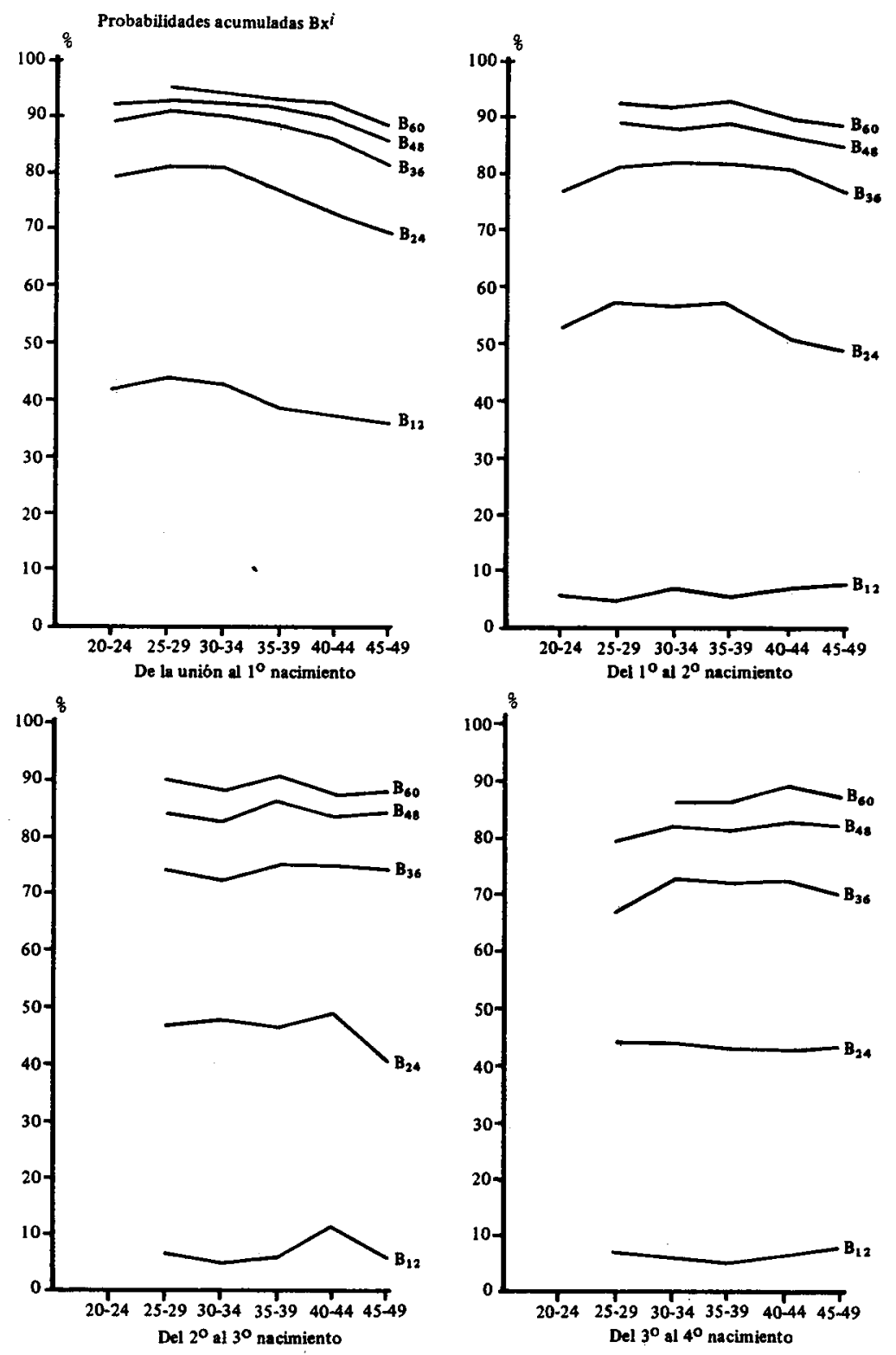
Probabilidad acumulada (Bx $l$ )
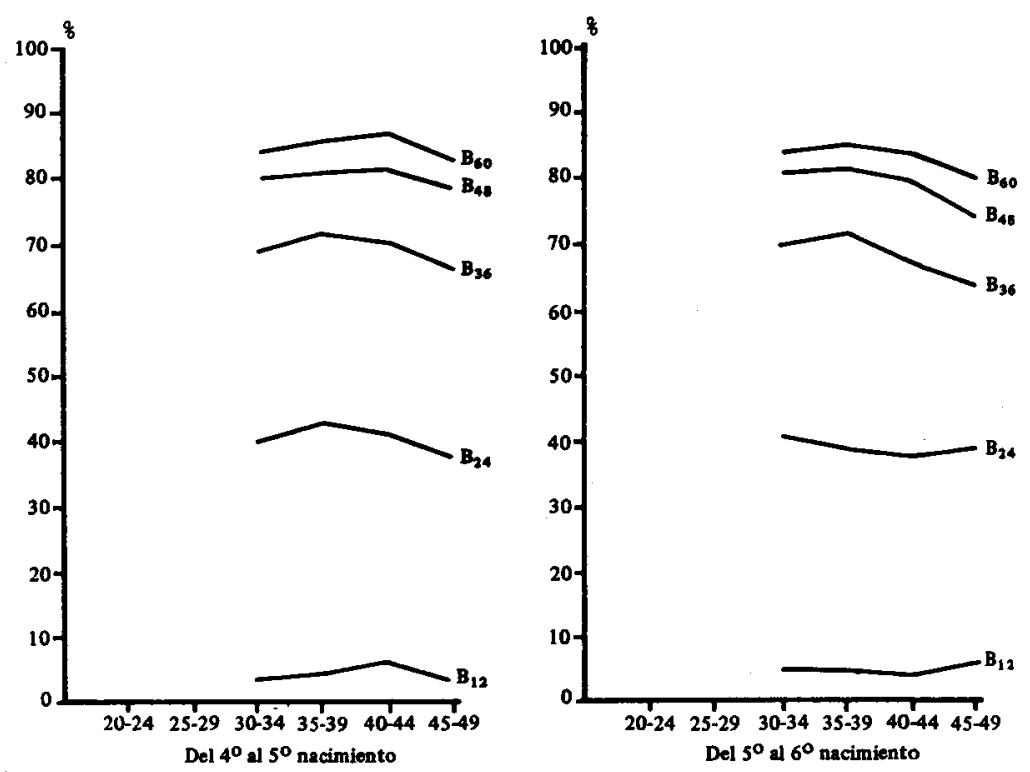

Fuente: Cuadro A.2 
GRÁFICA A. 4

Mediana del intervalo de los nacimientos en meses por cohortes, controlando la edad

de las mujeres en el nacimiento previo, para mujeres alguna vez unidas

Mediana en:
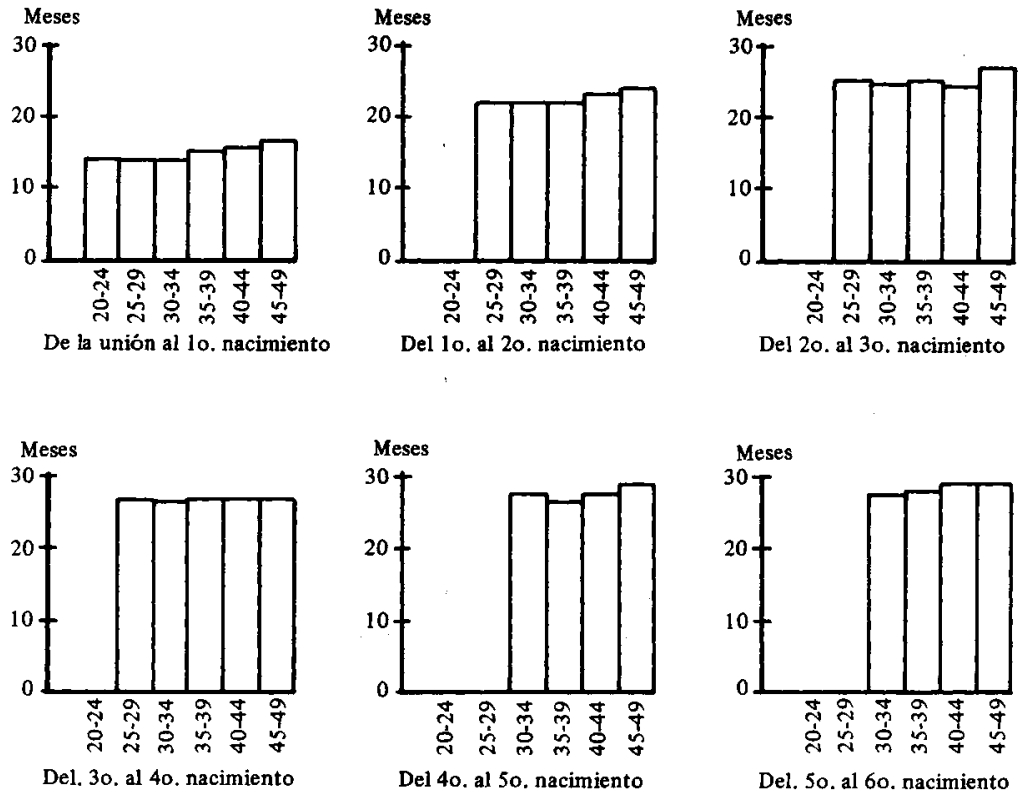

Del. 3o. al 4o. nacimiento

Del 4o. al 5o. nacimiento

Del. 5o. al 6o. nacimiento 
GRAFICA A.5

Proporciones acumuladas del intervalo de los nacimientos $P^{\text {in }}$ por cohortes, usando el método de truncamiento
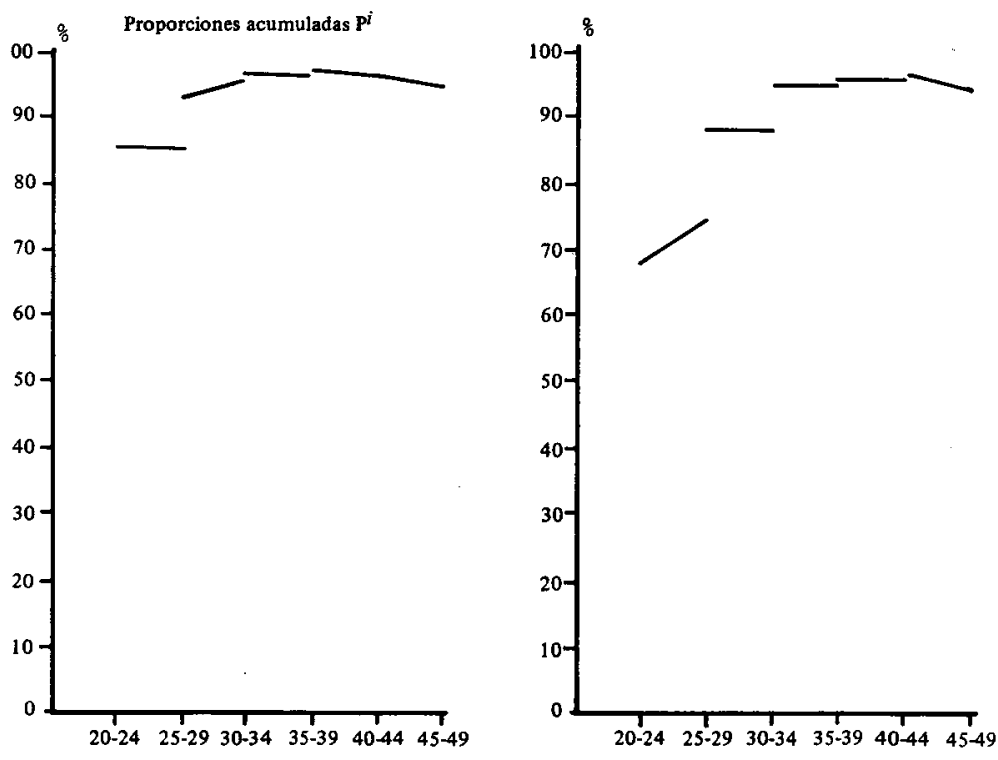

De la unión al $1^{\circ}$ nacimiento Del $1^{\circ}$ al $2^{\circ}$ nacimiento

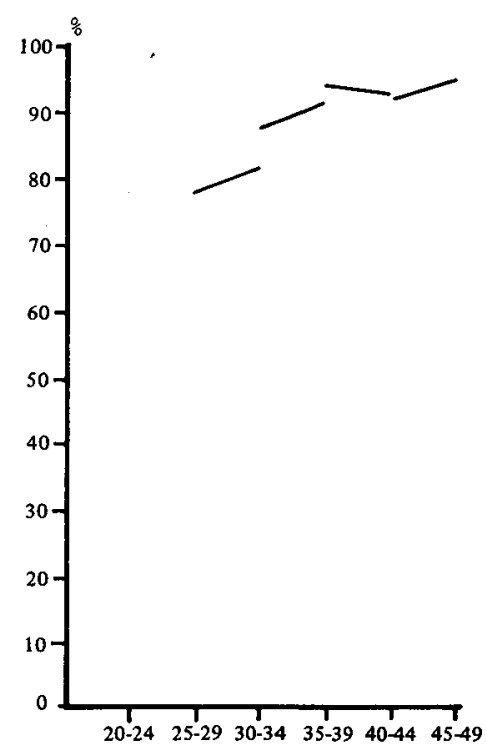
Del $2^{\circ}$ al $3^{\circ}$ nacimiento

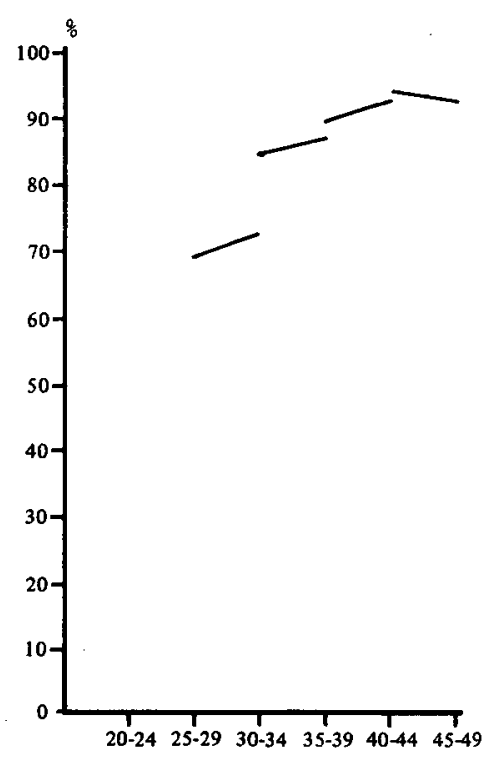
Del $3^{\circ}$ al $4^{\circ}$ nacimiento 
GRÁFICA A.5 (Continucción)

Proporciones acumuladas Pn
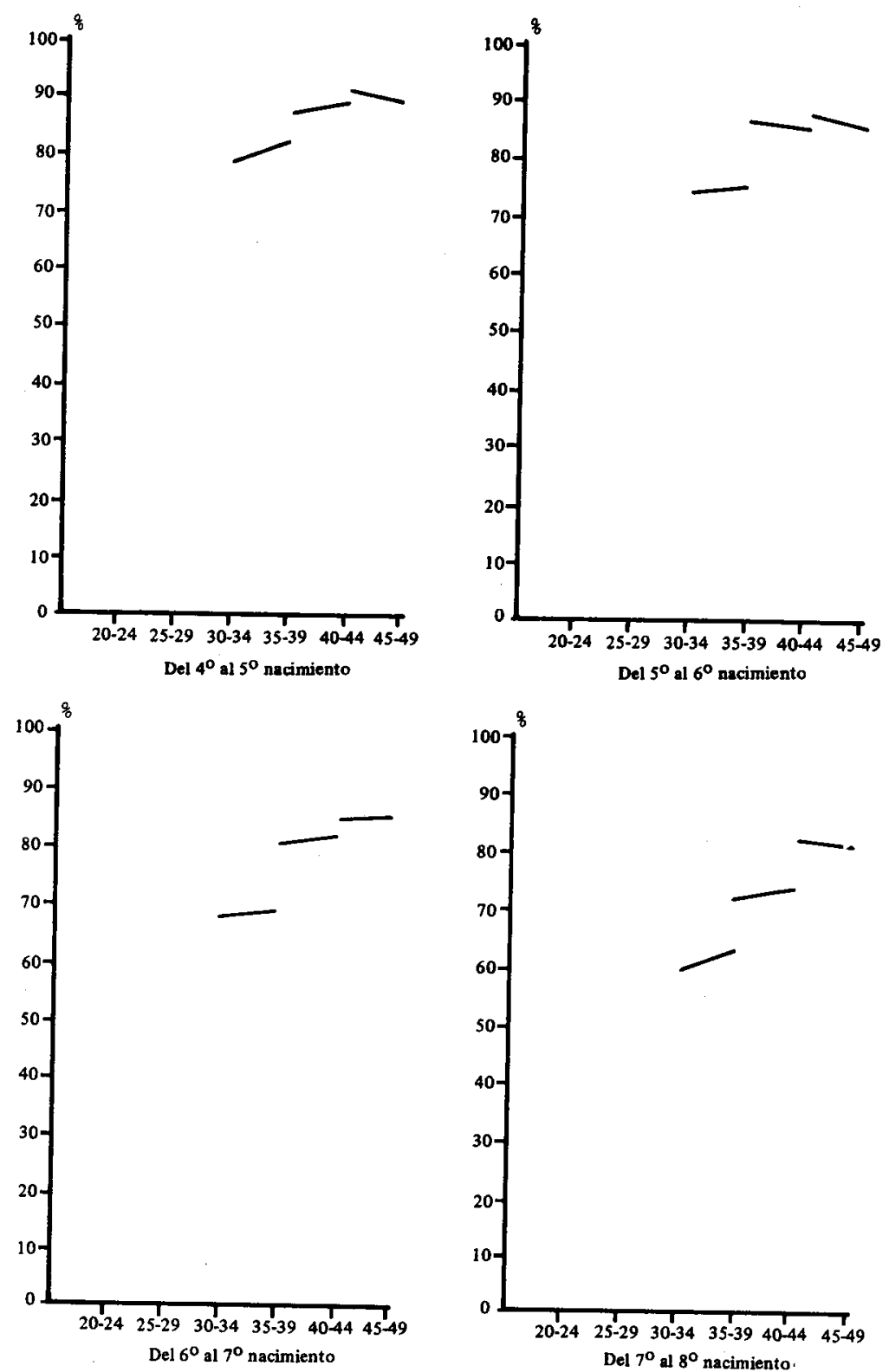
GRAFICAS A.s (Continuación)

Proporciones acumuladas $\mathrm{P}^{n}$

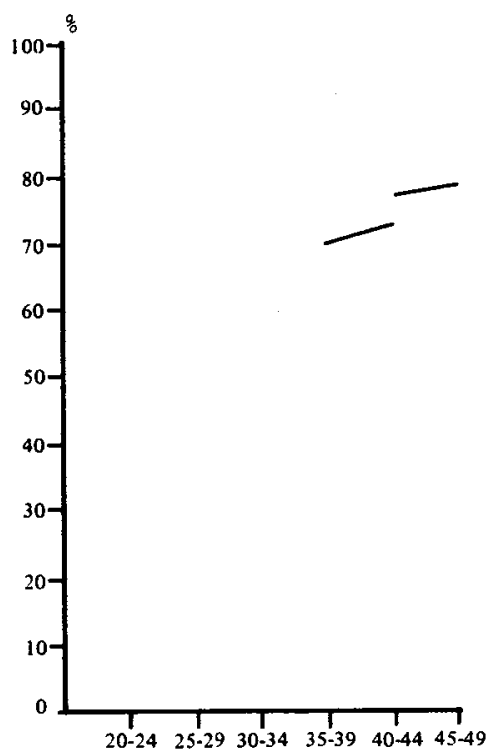

Del $8^{\circ}$ al $9^{\circ}$ nacimiento

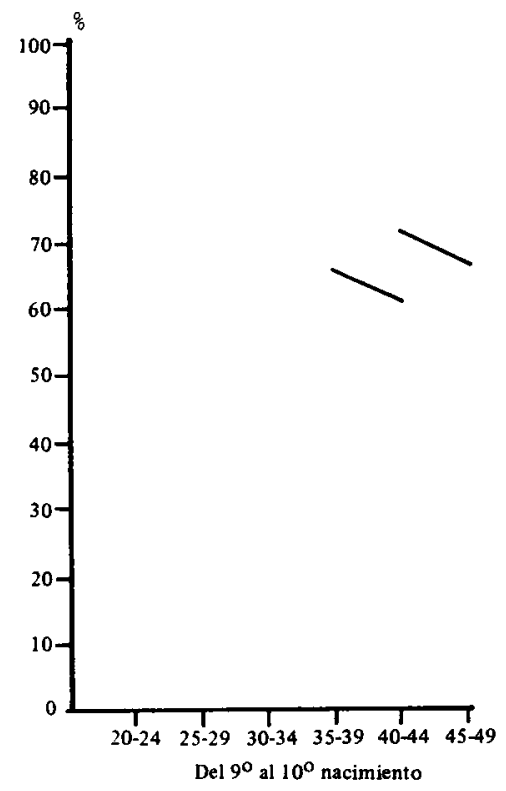


Probabilidades acumuladas $B_{60}$ para el intervalo de los nacimientos usando tablas de vida y proporciones acumuladas $P^{\prime}$ para el intervalo de los nacimientos según el método de truncamiento - cohortes truncadas en 5 años $B_{60}$ control de edad en el nacimiento previo
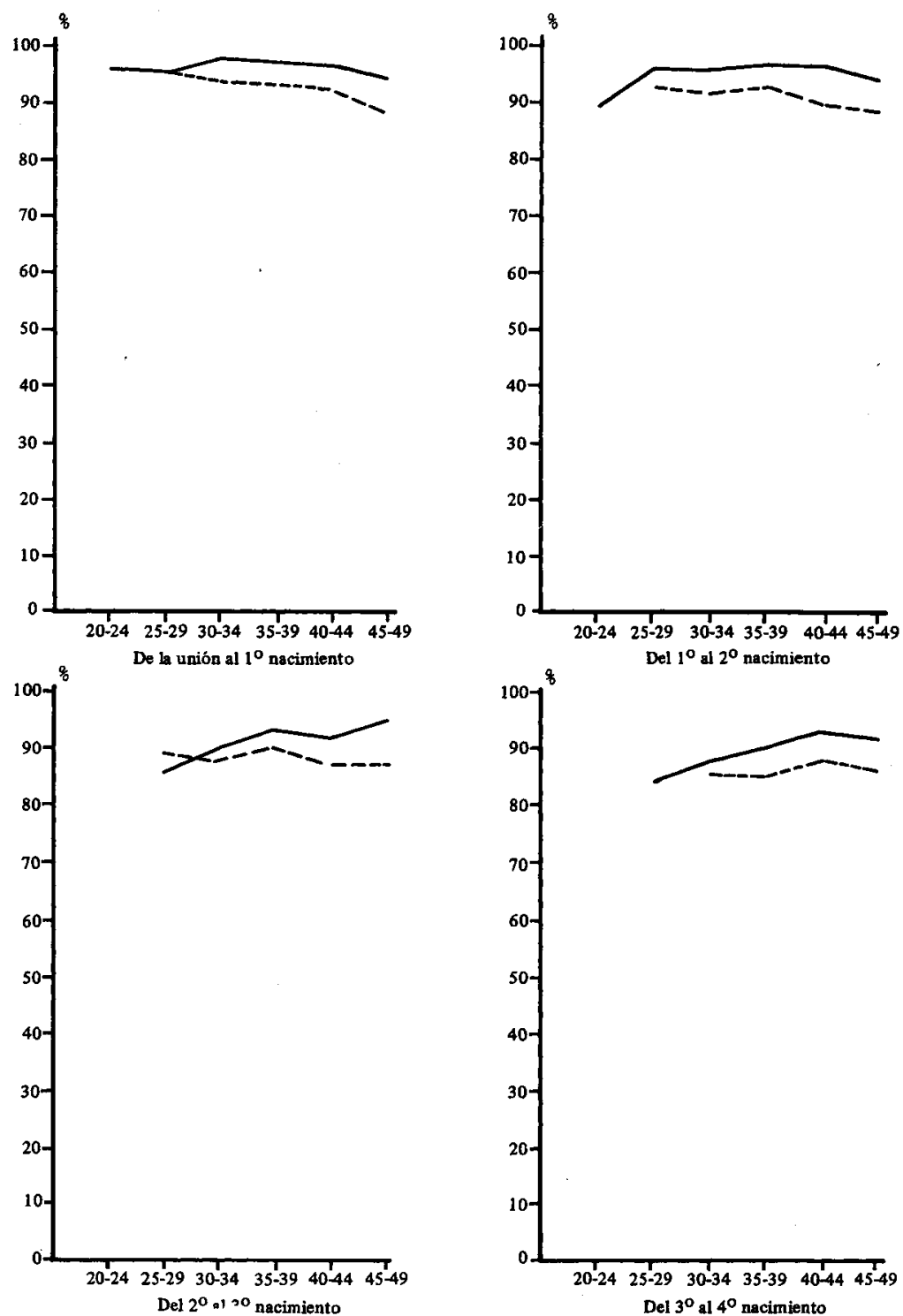

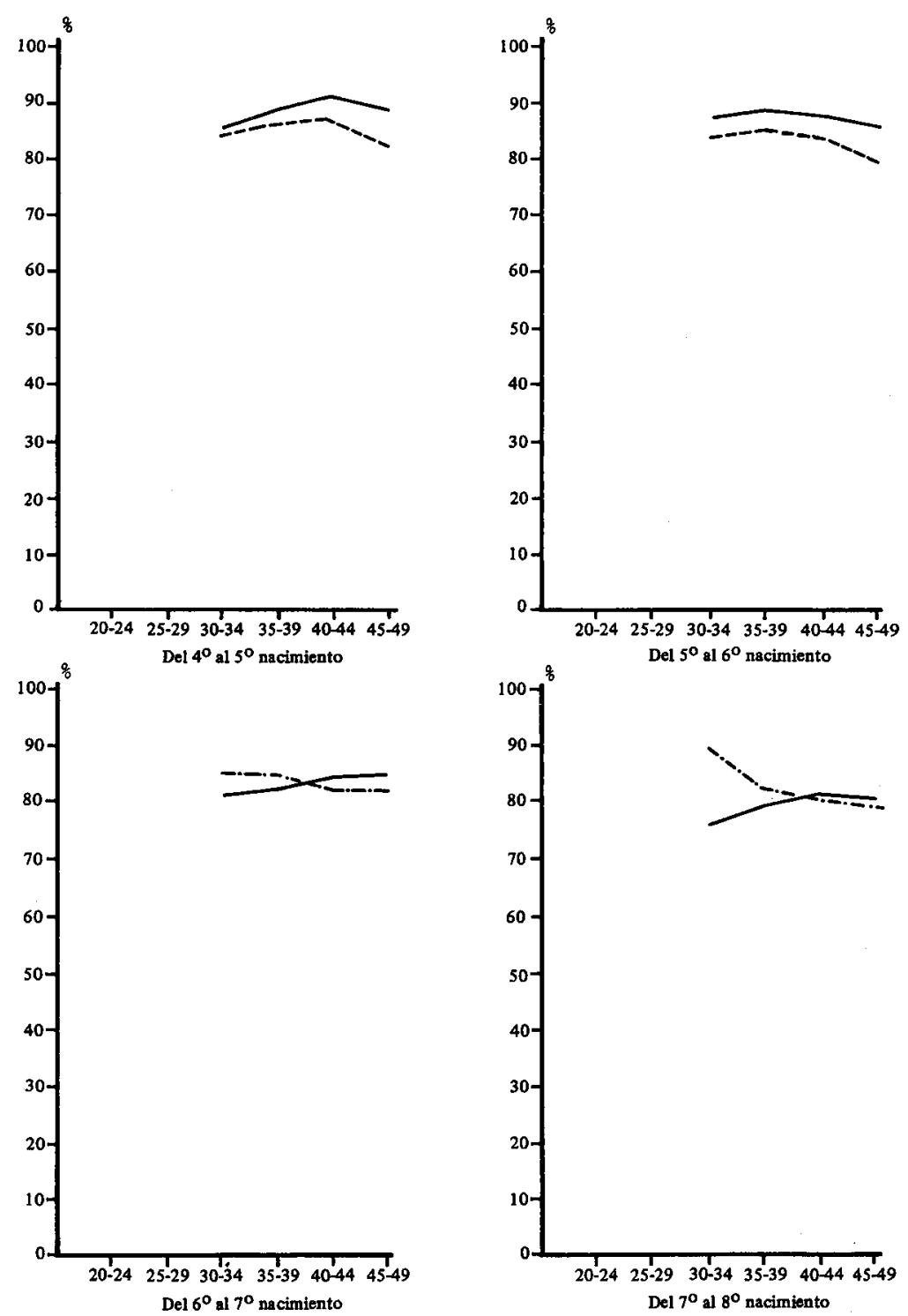
GRAFICA A.6 (Continuación)

_._. $\mathrm{P}^{\prime \prime}$ cohortes truncadas en 5 años
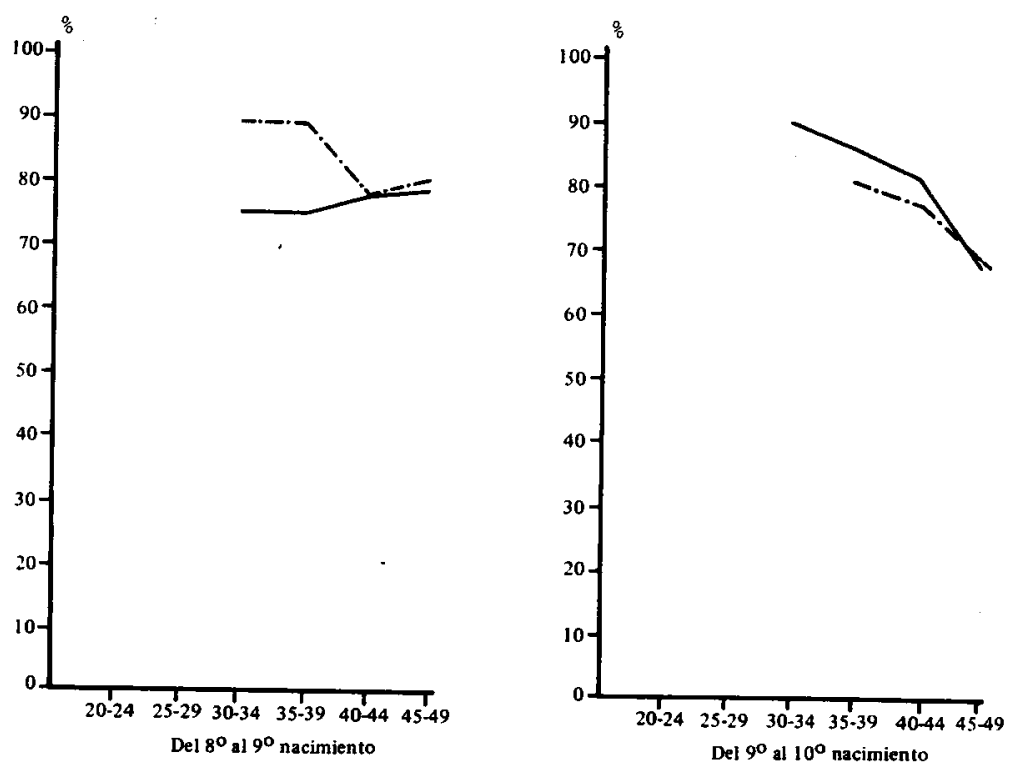

Fuente: Cuadro A.1, A.2 y A.4 
Cuadro A.1. Probabilidades acumuladas $\left(\mathrm{B}^{i} \mathrm{x}\right)$ del intervalo de los nacimientos para mujeres alguna vez unidas según duración de exposición al riesgo, 12, 24, 36 y 48 meses, por cohortes.

\begin{tabular}{|c|c|c|c|c|c|c|c|}
\hline $\mathrm{Bx}^{i}$ & $15-19$ & $20-24$ & $25-29$ & $30-34$ & $35-39$ & $40-44$ & $45-49$ \\
\hline $\begin{array}{l}1^{\mathrm{er}} \text { intervalo } \\
\text { de la unión al } \\
1^{\text {er nac. }}\end{array}$ & $(452)$ & (1029) & $(1095)$ & $(942)$ & $(879)$ & (669) & $(559)$ \\
\hline $\begin{array}{c}\mathbf{B}_{12} \\
\mathbf{B}_{24} \\
\mathbf{B}_{36} \\
\mathbf{B}_{48} \\
\mathbf{B}_{60} \\
\text { Mediana }\end{array}$ & $\begin{array}{l}.4141 \\
.8211 \\
.9105 \\
.9656 \\
.9656 \\
14.53\end{array}$ & $\begin{array}{l}.4238 \\
.7880 \\
.8984 \\
.9349 \\
.9613 \\
14.51\end{array}$ & $\begin{array}{l}.4445 \\
.8123 \\
.9079 \\
.9359 \\
.9559 \\
13.81\end{array}$ & $\begin{array}{l}.4343 \\
.8100 \\
.9004 \\
.9269 \\
.9396 \\
14.10\end{array}$ & $\begin{array}{l}.3936 \\
.7747 \\
.8851 \\
.9148 \\
.9334 \\
15.35\end{array}$ & $\begin{array}{l}.3812 \\
.7348 \\
.8651 \\
.9004 \\
.9220 \\
16.03\end{array}$ & $\begin{array}{l}.3703 \\
.6995 \\
.8193 \\
.8587 \\
.8839 \\
16.73\end{array}$ \\
\hline $\begin{array}{l}2^{\circ} \text { intervalo } \\
\text { del } 1^{\circ} \text { al } \\
2^{\circ} \text { nac. }\end{array}$ & $(301$ & $(882)$ & $(1020)$ & $(908)$ & $(856)$ & $(645)$ & $(530)$ \\
\hline $\begin{array}{c}\mathrm{B}_{12} \\
\mathrm{~B}_{24} \\
\mathrm{~B}_{36} \\
\mathrm{~B}_{48} \\
\mathrm{~B}_{60} \\
\text { Mediana }\end{array}$ & $\begin{array}{l}.0873 \\
.5392 \\
.8365 \\
.9066 \\
.9066 \\
20.96\end{array}$ & $\begin{array}{l}.0625 \\
.5337 \\
.8024 \\
.8853 \\
.9408 \\
23.14\end{array}$ & $\begin{array}{l}.0498 \\
.5751 \\
.8177 \\
.8941 \\
.9289 \\
22.28\end{array}$ & $\begin{array}{l}.0707 \\
.5690 \\
.8199 \\
.8807 \\
.9187 \\
22.34\end{array}$ & $\begin{array}{l}.0598 \\
.5730 \\
.8209 \\
.8899 \\
.9288 \\
22.29\end{array}$ & $\begin{array}{l}.0729 \\
.5132 \\
.8047 \\
.8654 \\
.9016 \\
23.64\end{array}$ & $\begin{array}{l}.0793 \\
.4915 \\
.7731 \\
.8453 \\
.8835 \\
24.36\end{array}$ \\
\hline
\end{tabular}


Cuadro A.1. Probabilidades acumuladas $\left(\mathrm{B}^{i} \mathrm{x}\right)$ del intervalo de los nacimientos para mujeres alguna vez unidas según duración de exposición al riesgo, 12, 24, 36, 48 y 60 meses, por cohortes (continuación)

\begin{tabular}{|c|c|c|c|c|c|c|c|}
\hline $\mathrm{B}^{i} \mathrm{x}$ & $15-19$ & $20-24$ & $25-29$ & $30-34$ & 35.39 & $40-44$ & $45-4 \subseteq$ \\
\hline $\begin{array}{c}3^{\mathrm{er}} \text { inter } \\
\text { del } 2^{\circ} \text { al } \\
3^{\mathrm{er}} \text { nac. } \\
\mathrm{B}_{12} \\
\mathrm{~B}_{24} \\
\mathrm{~B}_{36} \\
\mathrm{~B}_{48} \\
\mathrm{~B}_{60} \\
\text { Mediana }\end{array}$ & $\begin{array}{c}(1231) \\
.0518 \\
.4195 \\
.7097 \\
- \\
- \\
27.33\end{array}$ & $\begin{array}{c}(601) \\
.0519 \\
.4650 \\
.7919 \\
.9156 \\
- \\
25.28\end{array}$ & $\begin{array}{l}(899) \\
.0671 \\
.4708 \\
.7579 \\
.8658 \\
.9095 \\
25.22\end{array}$ & $\begin{array}{l}(861) \\
.0514 \\
.4782 \\
.7301 \\
.8325 \\
.8869 \\
25.04\end{array}$ & $\begin{array}{l}(821) \\
.0622 \\
.4611 \\
.7518 \\
.8649 \\
.9069 \\
.25 .61\end{array}$ & $\begin{array}{l}(625) \\
.0576 \\
.4823 \\
.7448 \\
.8345 \\
.8746 \\
24.81\end{array}$ & $\begin{array}{l}(500) \\
.0621 \\
.4528 \\
.7435 \\
.8421 \\
.8785 \\
25.95\end{array}$ \\
\hline $\begin{array}{l}4^{\circ} \text { interv } \\
\text { del } 3^{\circ} \text { al } \\
4^{\circ} \text { nac. }\end{array}$ & (26) & $(330)$ & $(702)$ & $(756)$ & (774) & $(575)$ & \\
\hline $\begin{array}{c}\mathbf{B}_{12} \\
\mathbf{B}_{24} \\
\mathbf{B}_{36} \\
\mathbf{B}_{48} \\
\mathbf{B}_{60} \\
\text { Mediana }\end{array}$ & $\begin{array}{c}.1111 \\
.2593 \\
- \\
- \\
- \\
-\end{array}$ & $\begin{array}{l}.0642 \\
.4468 \\
.7278 \\
.8128 \\
.8128 \\
26.27\end{array}$ & $\begin{array}{l}.0670 \\
.4425 \\
.7018 \\
.8245 \\
.8756 \\
26.66\end{array}$ & $\begin{array}{l}.0601 \\
.4396 \\
.7316 \\
.8246 \\
.8730 \\
26.48\end{array}$ & $\begin{array}{l}.0506 \\
.4301 \\
.7236 \\
.8194 \\
.8689 \\
26.86\end{array}$ & $\begin{array}{l}.0643 \\
.4226 \\
.7226 \\
.8251 \\
.8858 \\
27.10\end{array}$ & $\begin{array}{l}.075 \\
.437 \\
.700 \\
.821 \\
.870 \\
26.85\end{array}$ \\
\hline
\end{tabular}


Cuadro A.1. Probabilidades acumuladas $\left(\mathrm{B}^{i} \mathbf{x}\right)$ del intervalo de los nacimientos para mujeres alguna vez unidas según duración de exposición al riesgo, 12, 24, 36, 48 y 60 meses, por cohortes (continuación)

\begin{tabular}{|c|c|c|c|c|c|c|c|}
\hline $\mathrm{B}^{i_{\mathrm{X}}}$ & $15-19$ & $20-24$ & $25-29$ & $30-34$ & $35-39$ & $40-44$ & $45-49$ \\
\hline $\begin{array}{l}5^{\circ} \text { intervalo } \\
\text { del } 4^{\circ} \text { al } \\
5^{\circ} \text { nac. }\end{array}$ & (3) & $(127)$ & $(487)$ & $(640)$ & (695) & (539) & $(4391)$ \\
\hline $\begin{array}{c}\mathrm{B}_{12} \\
\mathrm{~B}_{24} \\
\mathrm{~B}_{36} \\
\mathrm{~B}_{48} \\
\mathrm{~B}_{60} \\
\text { Mediana }\end{array}$ & $\begin{array}{l}- \\
- \\
- \\
- \\
-\end{array}$ & $\begin{array}{c}.0758 \\
.4204 \\
.7102 \\
.9331 \\
- \\
27.30\end{array}$ & $\begin{array}{l}.0662 \\
.4060 \\
.7232 \\
.8322 \\
.8829 \\
27.56\end{array}$ & $\begin{array}{l}.0380 \\
.3891 \\
.7022 \\
.8125 \\
.8547 \\
27.99\end{array}$ & $\begin{array}{l}.0478 \\
.4339 \\
.7238 \\
.8157 \\
.8662 \\
26.74\end{array}$ & $\begin{array}{l}.0669 \\
.4152 \\
.7048 \\
.8161 \\
.8739 \\
27.51\end{array}$ & $\begin{array}{l}.0387 \\
.3827 \\
.6725 \\
.7962 \\
.8330 \\
28.86\end{array}$ \\
\hline $\begin{array}{l}6^{\circ} \text { intervalo } \\
\text { del } 5^{\circ} \text { al } \\
6^{\circ} \text { nac. }\end{array}$ & & $(42)$ & $(314)$ & (503) & $(606)$ & $(490)$ & $(390)$ \\
\hline $\begin{array}{c}\mathrm{B}_{12} \\
\mathrm{~B}_{24} \\
\mathrm{~B}_{36} \\
\mathrm{~B}_{48} \\
\mathrm{~B}_{60} \\
\text { Mediana }\end{array}$ & & $\begin{array}{c}.0000 \\
.2326 \\
- \\
- \\
- \\
-\end{array}$ & $\begin{array}{l}.0563 \\
.4285 \\
.6757 \\
.7786 \\
.8364 \\
27.47\end{array}$ & $\begin{array}{l}.0577 \\
.4302 \\
.7225 \\
.8359 \\
.8913 \\
26.86\end{array}$ & $\begin{array}{l}.0466 \\
.3897 \\
.7239 \\
.8208 \\
.8588 \\
27.96\end{array}$ & $\begin{array}{l}.0450 \\
.3760 \\
.6740 \\
.7997 \\
.8422 \\
29.05\end{array}$ & $\begin{array}{l}.0615 \\
.3974 \\
.6374 \\
.7447 \\
.8014 \\
29.10\end{array}$ \\
\hline
\end{tabular}


Cuadro A.1. Probabilidades acumuladas ( $\mathrm{B}^{i} \mathrm{x}$ ) del intervalo de los nacimientos para mujeres alguna vez unidas según duración de exposición al riesgo, 12, 24, 36, 48 y 60 meses, por cohortes (continuación)

\begin{tabular}{|c|c|c|c|c|c|c|c|}
\hline $\mathrm{B}^{i_{\mathrm{X}}}$ & 15.19 & 20.24 & 25.29 & $30-34$ & $35-39$ & 40.44 & $45-49$ \\
\hline \multicolumn{8}{|c|}{ COHORTES } \\
\hline $\begin{array}{l}7^{\circ} \text { interv } \\
\text { del } 6^{\circ} \text { al } \\
7^{\circ} \text { nac. }\end{array}$ & & (12) & $(153)$ & (373) & $(527)$ & $(432)$ & (343) \\
\hline $\begin{array}{c}\mathrm{B}_{12} \\
\mathrm{~B}_{24} \\
\mathrm{~B}_{36} \\
\mathrm{~B}_{48} \\
\mathrm{~B}_{60} \\
\text { Mediana }\end{array}$ & & $\begin{array}{c}.0000 \\
- \\
- \\
- \\
-\end{array}$ & $\begin{array}{l}.0480 \\
.4984 \\
.7277 \\
.7731 \\
.8762 \\
24.08\end{array}$ & $\begin{array}{l}.0618 \\
.4058 \\
.6948 \\
.8073 \\
.8564 \\
27.91\end{array}$ & $\begin{array}{l}.0699 \\
.3954 \\
.6772 \\
.7909 \\
.8504 \\
28.45\end{array}$ & $\begin{array}{l}.0372 \\
.3615 \\
.6570 \\
.7830 \\
.8249 \\
29.62\end{array}$ & $\begin{array}{l}.0643 \\
.3450 \\
.6673 \\
.7623 \\
.8278 \\
29.77\end{array}$ \\
\hline $\begin{array}{l}8^{\circ} \text { interv } \\
\text { del } 7^{\circ} \text { al } \\
8^{\circ} \text { nac. }\end{array}$ & & (4) & (63) & (253) & (423) & $(366)$ & $(301)$ \\
\hline $\begin{array}{c}\mathbf{B}_{12} \\
\mathbf{B}_{24} \\
\mathbf{B}_{36} \\
\mathbf{B}_{48} \\
\mathbf{B}_{60} \\
\text { Mediana }\end{array}$ & & $\begin{array}{l}- \\
- \\
- \\
- \\
-\end{array}$ & $\begin{array}{c}.0638 \\
.5338 \\
.6871 \\
.7914 \\
\dot{-} \\
23.14\end{array}$ & $\begin{array}{l}.0493 \\
.4486 \\
.7809 \\
.8734 \\
.8993 \\
25.86\end{array}$ & $\begin{array}{l}.0363 \\
.3767 \\
.6778 \\
.7853 \\
.8320 \\
28.91\end{array}$ & $\begin{array}{l}.0495 \\
.3867 \\
.6875 \\
.7789 \\
.8085 \\
28.52\end{array}$ & $\begin{array}{l}.0301 \\
.2809 \\
.5925 \\
.7392 \\
.7973 \\
32.44\end{array}$ \\
\hline
\end{tabular}


Cuadro A.1. Probabilidades acumuladas $\left(\mathrm{B}^{i} \mathrm{x}\right.$ ) del intervalo de los nacimientos para mujeres alguna vez unidas según duración de exposición al riesgo, 12, 24, 36 y 60 meses, por cohortes (conclusión)

\begin{tabular}{|c|c|c|c|c|c|c|c|}
\hline $\mathrm{B}^{i_{\mathrm{x}}}$ & $15-29$ & $20-24$ & 25.29 & $30-34$ & $35-39$ & $40-44$ & $45-49$ \\
\hline $\begin{array}{l}9^{\circ} \text { intervalo } \\
\text { del } 8^{\circ} \text { al } \\
9^{\circ} \text { nac. }\end{array}$ & & & (22) & $(151)$ & $(305)$ & (301) & (248) \\
\hline $\begin{array}{c}\mathbf{B}_{12} \\
\mathbf{B}_{24} \\
\mathbf{B}_{36} \\
\mathbf{B}_{48} \\
\mathbf{B}_{60} \\
\text { Mediana }\end{array}$ & & & $\begin{array}{c}.0606 \\
.8018 \\
- \\
- \\
19.11\end{array}$ & $\begin{array}{l}.0861 \\
.4287 \\
.7441 \\
.7841 \\
.8877 \\
26.71\end{array}$ & $\begin{array}{l}.0686 \\
.3773 \\
.6863 \\
.8070 \\
.8807 \\
28.76\end{array}$ & $\begin{array}{l}.0571 \\
.3836 \\
.6648 \\
.7374 \\
.7789 \\
28.97\end{array}$ & $\begin{array}{l}.0363 \\
.2996 \\
.5900 \\
.7339 \\
.7994 \\
32.28\end{array}$ \\
\hline \multicolumn{8}{|c|}{ COHORTES } \\
\hline $\begin{array}{l}10^{\circ} \text { intervalo } \\
\text { del } 9^{\circ} \text { al } \\
10^{\circ} \text { nac. }\end{array}$ & & & (8) & (75) & $(215)$ & $(232)$ & (207) \\
\hline $\begin{array}{l}B_{12} \\
B_{24} \\
B_{36} \\
B_{48} \\
B_{60} \\
\text { Mediana }\end{array}$ & & & $\begin{array}{c}.0000 \\
.2500 \\
.5000 \\
- \\
- \\
36.0\end{array}$ & $\begin{array}{c}.0841 \\
.4588 \\
.6837 \\
- \\
- \\
26.20\end{array}$ & $\begin{array}{l}.0640 \\
.4135 \\
.6632 \\
.7810 \\
.8114 \\
28.16\end{array}$ & $\begin{array}{l}.0751 \\
.3882 \\
.6250 \\
.7174 \\
.7758 \\
29.66\end{array}$ & $\begin{array}{l}.0573 \\
.2972 \\
.5357 \\
.6565 \\
.6855 \\
33.41\end{array}$ \\
\hline
\end{tabular}

Fuente: Cálculos elaborados con base en la Encuesta Mexicana de Fecundidad 1976-1977. 
Cuadro A.2. Probabilidades acumuladas ( $\mathrm{B}^{i} \mathrm{x}$ ) del intervalo de los nacimientos controlando la edad de las mujeres en el nacimiento previo para mujeres alguna vez unidas según duración de exposición al riesgo $12,24,36,48$ y 60 meses, por cohortes

\begin{tabular}{|c|c|c|c|c|c|c|c|}
\hline $\mathrm{B}^{i_{\mathrm{X}}}$ & $15-19$ & $20-24$ & $\begin{array}{c}\mathrm{COHO} \\
25-29\end{array}$ & $30-34$ & $35-39$ & $40-44$ & $45-49$ \\
\hline \multicolumn{8}{|c|}{$\begin{array}{l}1^{\text {er }} \text { intervalo } \\
\text { de la unión } \\
\text { al } 1^{\text {er nac. }}\end{array}$} \\
\hline $\begin{array}{c}\mathbf{B}_{12} \\
\mathbf{B}_{24} \\
\mathbf{B}_{36} \\
\mathbf{B}_{48} \\
\mathbf{B}_{60} \\
\text { Mediana }\end{array}$ & $\begin{array}{c}.4093 \\
.8352 \\
- \\
- \\
- \\
14.55\end{array}$ & $\begin{array}{c}.4210 \\
.7904 \\
. .8932 \\
.9229 \\
- \\
14.57\end{array}$ & $\begin{array}{l}.4414 \\
.8111 \\
.9071 \\
.9331 \\
.9528 \\
13.90\end{array}$ & $\begin{array}{l}.4328 \\
.8097 \\
.8995 \\
.9262 \\
.9381 \\
14.14\end{array}$ & $\begin{array}{l}.3860 \\
.7719 \\
.8836 \\
.9136 \\
.9324 \\
15.54\end{array}$ & $\begin{array}{l}.3737 \\
.7316 \\
.8634 \\
.8988 \\
.9205 \\
16.23\end{array}$ & $\begin{array}{l}.3634 \\
.6961 \\
.8173 \\
.8572 \\
.8855 \\
16.93\end{array}$ \\
\hline \multicolumn{8}{|c|}{$\begin{array}{l}2^{\circ} \text { intervalo } \\
\text { del } 1^{\circ} \text { al } \\
2^{\circ} \text { nac. }\end{array}$} \\
\hline $\begin{array}{c}\mathrm{B}_{12} \\
\mathrm{~B}_{24} \\
\mathrm{~B}_{36} \\
\mathrm{~B}_{48} \\
\mathrm{~B}_{60} \\
\text { Mediana }\end{array}$ & $\begin{array}{c}.0538 \\
.4542 \\
- \\
- \\
- \\
-\end{array}$ & $\begin{array}{c}.0622 \\
.5293 \\
.7716 \\
- \\
- \\
23.25\end{array}$ & $\begin{array}{l}.0500 \\
.5744 \\
.8130 \\
.8897 \\
.9244 \\
22.30\end{array}$ & $\begin{array}{l}.0708 \\
.5671 \\
.8190 \\
.8794 \\
.9169 \\
22.38\end{array}$ & $\begin{array}{l}.0593 \\
.5727 \\
.8206 \\
.8893 \\
.9289 \\
22.30\end{array}$ & $\begin{array}{l}.0737 \\
.5132 \\
.8055 \\
.8652 \\
.9019 \\
23.64\end{array}$ & $\begin{array}{l}.0783 \\
.4894 \\
.7743 \\
.8472 \\
.8884 \\
24.45\end{array}$ \\
\hline
\end{tabular}


Cuadro A.2. Probabilidades acumuladas ( $\mathrm{B}^{i_{\mathrm{X}}}$ ) del intervalo de los nacimientos controlando la edad de las mujeres en el nacimiento previo para mujeres alguna vez unidas según duración de exposición al riesgo $12,24,36,48$ y 60 meses; por cohortes (continuación)

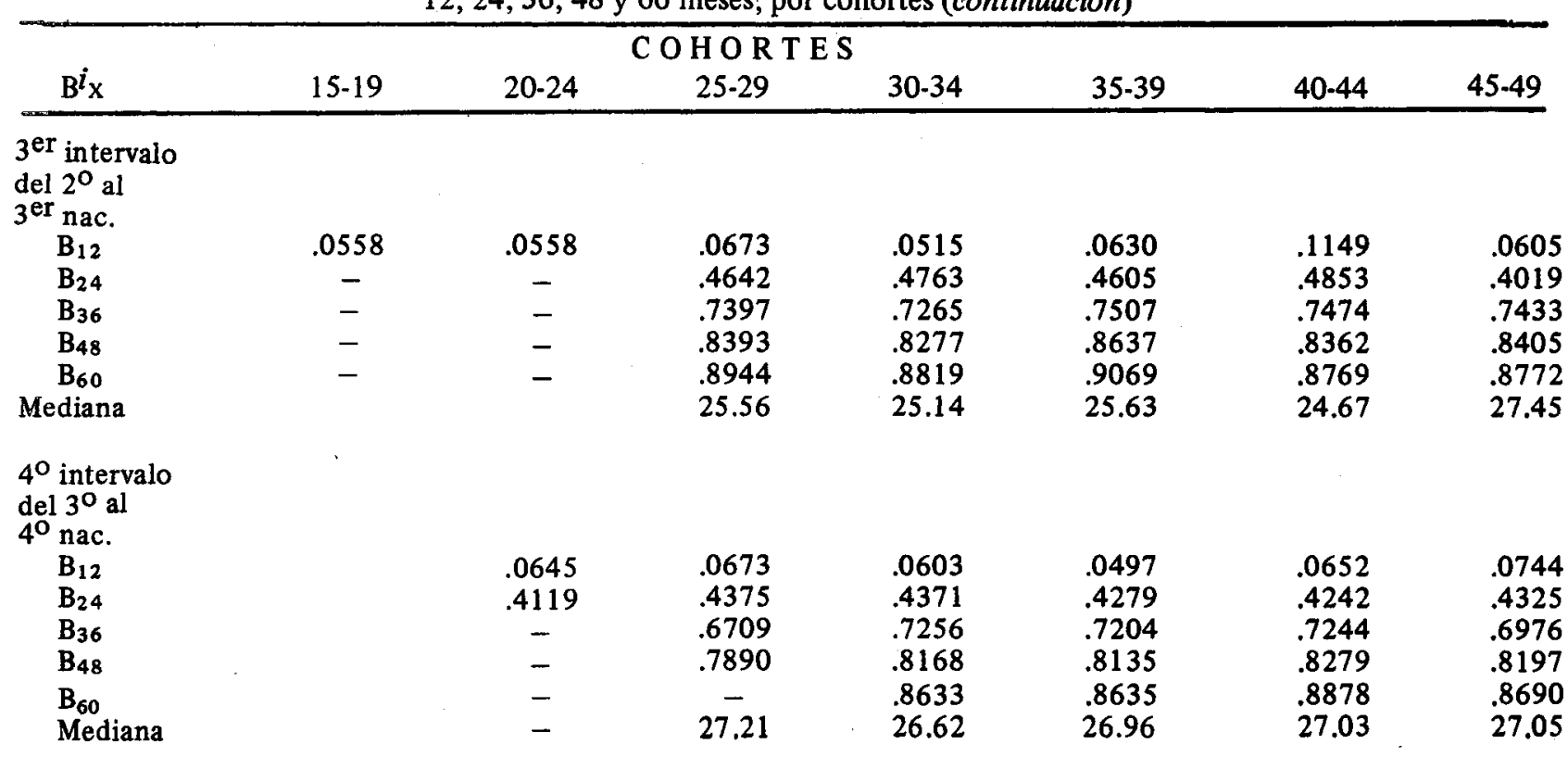


Cuadro A.2. Probabilidades acumuladas $\left(\mathrm{B}^{i} \mathrm{x}\right.$ ) del intervalo de los nacimientos controlando la edad de las mujeres en el nacimiento previo para mujeres alguna vez unidas según duración de exposición al riesgo

$12,24,36,48$ y 50 meses, por cohortes (conclusión)

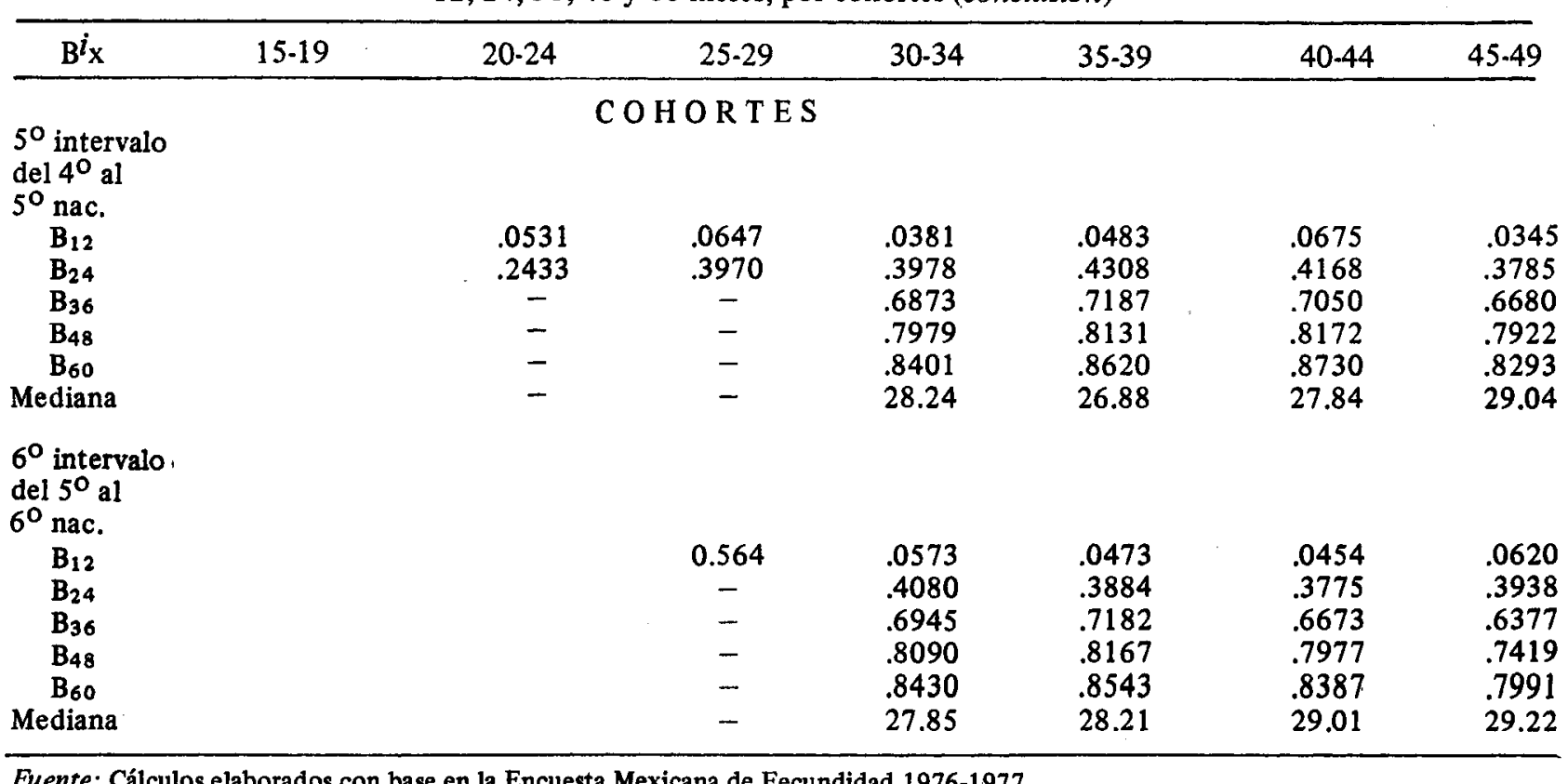

Fuente: Cálculos elaborados con base en la Encuesta Mexicana de Fecundidad 1976-1977. 
Cuadro A.3. Número absoluto de mujeres en cada estadio del proceso de formación de la familia por cohortes quinquenales, usando el Método de Truncamients.

\begin{tabular}{|c|c|c|c|c|c|c|c|c|c|c|c|}
\hline \multirow[t]{2}{*}{$\begin{array}{l}\text { Experiencia } \\
\text { acumulada } \\
\text { de cohortes } \\
\text { truncadas }\end{array}$} & \multicolumn{11}{|c|}{ Número de mujeres en cada estadio del proceso de formación de la familia } \\
\hline & A la unión & ler. nac. & 20. nac. & 3er. nac. & 40. nac. & 5o. nac. & 60. nac. & 7o. nac. & 80. nac. & 90. nac. & 10o. nac. \\
\hline$C_{t} 15-19$ & 447 & 296 & 121 & 26 & 3 & - & - & - & & & \\
\hline$c^{\prime} 20-24$ & 401 & 243 & 87 & 25 & 5 & - & - & - & - & - & - \\
\hline$C_{t} 20-24$ & 1024 & 877 & 598 & 327 & 126 & 42 & 12 & 4 & - & - & - \\
\hline$c^{25-29}$ & 856 & 730 & 545 & 320 & 133 & 52 & 14 & 2 & 2 & - & - \\
\hline$C_{t} 25-29$ & 1088 & 1013 & 892 & 695 & 481 & 308 & 150 & 63 & 22 & 8 & 3 \\
\hline$c^{1} 30-34$ & 886 & 847 & 747 & 610 & 444 & 279 & 170 & 75 & 21 & 4 & 2 \\
\hline$c_{t} 30-34$ & 939 & 905 & 858 & 753 & 637 & 501 & 373 & 253 & 151 & 75 & 38 \\
\hline$c^{1} 35-39$ & 855 & 820 & 782 & 715 & 622 & 510 & 387 & 267 & 169 & 84 & 31 \\
\hline$c_{t} 35-39$ & 868 & 845 & 811 & 765 & 686 & 597 & 520 & 416 & 301 & 211 & 139 \\
\hline$c^{t} 40-44$ & 656 & 632 & 607 & 563 & 522 & 464 & 400 & 327 & 243 & 177 & 109 \\
\hline$C_{t} 40-44$ & 661 & 637 & 617 & 568 & 534 & 485 & 428 & 362 & 298 & 231 & 166 \\
\hline$c^{2} 45-49$ & 552 & 522 & 493 & 468 & 433 & 385 & 333 & 284 & 231 & 182 & 123 \\
\hline C $45-49$ & 553 & 524 & 496 & 471 & 435 & 386 & 339 & 297 & 244 & 204 & 140 \\
\hline Total & 5580 & 5097 & 4393 & 3605 & 2902 & 2319 & 1822 & 1395 & 1016 & 729 & 486 \\
\hline
\end{tabular}

Fuente: Cálculos elaborados con base en la Encuesta Mexicana de Fecundidad 1976-1977. 
Cuadro A.4. Proporción acumulada del intervalo de los nacimientos Pn por cohortes quinquenales, usando el Método de Truncamiento.

\begin{tabular}{|c|c|c|c|c|c|c|c|c|c|c|}
\hline \multirow{2}{*}{$\begin{array}{l}\text { Experiencia } \\
\text { acumulada } \\
\text { de cohortes } \\
\text { truncadas }\end{array}$} & \multicolumn{10}{|c|}{ Proporción acumulada de mujeres con nacimiento ${ }^{n}$ que continúa al $n+1(P n)$} \\
\hline & $\begin{array}{l}\text { De la unión } \\
\text { al ler. nac. }\end{array}$ & $\begin{array}{l}\text { Del lo. al } \\
\text { 2o. nac. }\end{array}$ & $\begin{array}{l}\text { Del 2o. al } \\
\text { 3er. nac. }\end{array}$ & $\begin{array}{c}\text { Del 3o. al } \\
\text { 4o, nac. }\end{array}$ & $\begin{array}{l}\text { Del 4o. al } \\
\text { 5o. nac. }\end{array}$ & $\begin{array}{c}\text { Del 5o. al } \\
60 . \text { nac. }\end{array}$ & $\begin{array}{c}\text { Del 60. al } \\
\text { 7o. nac. }\end{array}$ & $\begin{array}{l}\text { Del 7o. al } \\
\text { 80. nac. }\end{array}$ & $\begin{array}{l}\text { Del 80. al } \\
90 . \text { nac. }\end{array}$ & $\begin{array}{l}\text { Del 90. al } \\
100 \text {. nac. }\end{array}$ \\
\hline$C_{t} 15-19$ & .6622 & 4088 & .2149 & .1154 & - & - & - & - & - & - \\
\hline$c^{\mathbf{t}} 20-24$ & .6060 & .3580 & .2874 & .2000 & - & - & - & - & - & - \\
\hline$c_{t} 20-24$ & .8564 & 6819 & .5468 & .3833 & .3300 & 2857 & .3333 & - & - & - \\
\hline$c^{2} 25-29$ & .8528 & .7466 & .5872 & 4156 & 3910 & 2692 & 1429 & 1.0000 & - & - \\
\hline$C_{t^{25-29}}$ & .9311 & .8805 & .7791 & .6921 & .6403 & .4870 & .4200 & .3492 & .3636 & .3750 \\
\hline$C^{2} 30-34$ & .9560 & 8819 & .8166 & .7279 & .6284 & .6093 & .4412 & . 2800 & 1905 & .5000 \\
\hline$c_{t} 30-34$ & .9638 & .9481 & .8776 & .8459 & .7865 & .7445 & .6783 & .5968 & .4967 & .5067 \\
\hline$C^{l} 35-39$ & .9591 & .9537 & .9143 & .8699 & .8199 & .7588 & .6899 & .6330 & .4970 & .3690 \\
\hline$c_{t} 35-39$ & .9735 & .9598 & .9433 & .8967 & .8703 & .8710 & .8000 & .7236 & .7010 & .6588 \\
\hline$c^{t} 40-44$ & .9634 & 9604 & .9275 & .9272 & .8889 & .8621 & .8175 & .7431 & .7284 & .6158 \\
\hline$c_{t}{ }^{40-44}$ & .9637 & .9686 & 9206 & .9401 & .9082 & .8825 & .8458 & .8232 & .7752 & .7186 \\
\hline$C^{2} 45-49$ & .9456 & .9444 & .9493 & .9252 & .8891 & .8649 & .8528 & .8134 & .7879 & .6758 \\
\hline C $45-49$ & .9476 & .9466 & .9496 & .9236 & .8874 & .8782 & .8761 & .8215 & .8361 .6863 & $\begin{array}{ll}3 & .6863\end{array}$ \\
\hline Total & .9134 & .8619 & .8206 & .8050 & .7957 & .7857 & .7656 & .7283 & .7175 & .6663 \\
\hline
\end{tabular}

Fuente: Cuadro A.3. 
Cuadro A.5. Media del intervalo de los nacimientos en meses por cohortes, usando el Método de Truncamiento.

\begin{tabular}{|c|c|c|c|c|c|c|c|c|c|c|}
\hline \multirow{2}{*}{$\begin{array}{l}\text { Experiencia } \\
\text { acumulada } \\
\text { de cohortes } \\
\text { truncadas }\end{array}$} & \multirow[b]{2}{*}{$\begin{array}{l}\text { De la unión } \\
\text { al ler. nac. }\end{array}$} & \multirow[b]{2}{*}{$\begin{array}{l}\text { Del 10. al } \\
20 . \text { nac. }\end{array}$} & \multicolumn{4}{|c|}{ Mediana del intervalo en meses } & \multirow[b]{2}{*}{$\begin{array}{c}\text { Del 6o. al } \\
70 . \text { nac. }\end{array}$} & \multirow[b]{2}{*}{$\begin{array}{l}\text { Del 7o. al } \\
\text { 8o. nac. }\end{array}$} & \multirow[b]{2}{*}{$\begin{array}{c}\text { Del 80. al } \\
90 . \text { nac. }\end{array}$} & \multirow[b]{2}{*}{$\begin{array}{l}\text { Del 9o. al } \\
100 \text {. nac. }\end{array}$} \\
\hline & & & $\begin{array}{l}\text { Del 2o. al } \\
\text { 3er. nac. }\end{array}$ & $\begin{array}{l}\text { Del 3o. al } \\
\text { 4o. nac. }\end{array}$ & $\begin{array}{l}\text { Del 4o. al } \\
\text { 5o. nac. }\end{array}$ & $\begin{array}{l}\text { Del 5o. al } \\
60 . \text { nac. }\end{array}$ & & & & \\
\hline$C_{t} 15-19$ & 13.38 & 19.16 & 17.88 & 12.33 & - & - & - & - & - & - \\
\hline$c^{l} 20-24$ & 14.78 & 18.18 & 16.52 & 17.80 & - & - & - & - & - & - \\
\hline $\mathrm{C}_{\mathrm{t}} 20-24$ & 15.61 & 22.10 & 21.76 & 20.65 & 21.69 & 21.08 & 17.75 & - & - & - \\
\hline$C^{l} 25-29$ & 15.28 & 21.73 & 21.20 & 20.09 & 20.31 & 18.86 & 19.00 & 15.00 & - & - \\
\hline$C_{t} 25-29$ & 15.88 & 23.89 & 24.62 & 24.33 & 24.21 & 22.42 & 21.49 & 19.04 & 16.50 & 28.33 \\
\hline C $30-34$ & 17.42 & 23.49 & 24.57 & 24.01 & 22.97 & 21.48 & 20.65 & 20.43 & 8.25 & 17.00 \\
\hline$C_{t} 30-34$ & 17.80 & 25.18 & 27.04 & 26.81 & 26.48 & 25.12 & 25.35 & 22.89 & 21.67 & 19.89 \\
\hline $\mathrm{C}^{\mathrm{t}} 35-39$ & 19.34 & 25.80 & 26.37 & 26.85 & 25.06 & 26.42 & 23.60 & 23.60 & 22.11 & 20.74 \\
\hline$C_{t} 35-39$ & 19.80 & 26.10 & 28.05 & 28.91 & 27.47 & 28.65 & 26.90 & 26.31 & 25.40 & 24.39 \\
\hline$c^{2} 40-44$ & 20.46 & 27.43 & 26.96 & 28.82 & 27.23 & 27.78 & 27.41 & 28.14 & 22.53 & 23.04 \\
\hline$c_{t} 40-44$ & 20.76 & 28.78 & 27.47 & 29.98 & 28.00 & 30.27 & 28.52 & 27.46 & 26.46 & 25.33 \\
\hline$c^{t} 45-49$ & 22.99 & 28.58 & 29.97 & 29.63 & 29.93 & 30.31 & 28.63 & 29.66 & 29.07 & 26.16 \\
\hline C $45-49$ & 24.10 & 28.95 & 30.15 & 29.94 & 30.00 & 31.00 & 29.43 & 31.03 & 31.23 & 27.99 \\
\hline Total & 18.13 & 35.43 & 26.32 & 27.67 & 27.25 & 28.18 & 27.31 & 27.11 & 26.89 & 25.42 \\
\hline
\end{tabular}

Fuente: Cuadro A.3. 
Bibliografía

BRASs, W., The Analysis of Maternity Histories to Detect Changes in Fertility, Budapest, Consejo Económico y Social de las Naciones Unidas, Nueva York, 1971.

BooTH, H., The estimation of Fertility from Incomplete Cohort Data by Means of the Transformed Gompertz Model, tesis para obtener el grado de doctar en Demografía, Universidad de Londres, Inglaterra, 1979.

HoBCRAFT, J. y RoDríguez, G., Methodological Issues in Life Table Analysis of Birth Histories, documento presentado al seminario sobre el Análisis de Historias de la Maternidad, organizado por la IUSSP, CCAF, WFS y la Escuela de Higiene y Medicina Tropical de Londres, 9-11 de abril, Londres, Inglaterra, 1980.

"Life Table Analysis of Birth Intervals in Colombia: An Illustrative Analysis", Encuesta Mundial de Fecundidad, Instituto Internacional de Estadística, Londres, Inglaterra, 1980.

JUÁREZ, F., Análisis del proceso de formación de familias en México, documento presentado al Segundo Congreso Nacional de Demografia, México, 1980.

Ordorica, M. y Potter, J. E., An Evahuation of the Demographic Data Collected in the Mexican Fertility Survey, 1980 (mimeo.).

Poot, J., "Family-Building in a Canadian City: an Analysis of the Timing and Spacing of Pregnancies", Population Studies, vol. 32, núm. 3, Inglaterra, noviembre 1978.

PotTer, J. E., "Problems in Using Birth-History Analysis to Estimate Trends in Fertility", Population Studies, vol. 31, núm. 2, Inglaterra, 1977.

RYDER, N. B., Seminar on Tabulation and an Analysis of Survey Data, Encuesta Mundial de Fecundidad del Instituto Internacional de Estadística, Londres, Inglaterra, 1974.

Smith, D. P., "Life Table Analysis", Technical Bulletins, Encuesta Mundial de Fecundidad del Instituto Internacional de Estadística, Londres, Inglaterra, 1980.

Stoto, M. y MENKEN, J. A., Birth Intervals in the U.S., 1973: A New Model Applied to Data from the National Survey of Family Growth, Cycle I, documento presentado a la reunión anual de la Asociación de Población de América, EEUU, 1977.

TUKEY, J. W., Exploratory Data Analysis, Addison-Wesley, EEuU, 1977.

Young, C. M., "Spacing of Children and Changing Pacterns of Child- 
bearing", Journal of Biosocial Science, vol. 9, núm. 2, Inglaterra, abril 1977.

ZABA, B., Use of the Relational Gompertz in Analysing Fertility Data Collected in Retrospective Surveys, 1978 (mimeo.). 